In Situ

Revue des patrimoines
In Situ

Revue des patrimoines

$17 \mid 2011$

Les patrimoines de l'enseignement supérieur

\title{
Le campus et le C.H.U de Bordeaux 1959-2005
}

\section{Marc Saboya}

\section{OpenEdition}

\section{Journals}

Édition électronique

URL : http://journals.openedition.org/insitu/6493

DOI : $10.4000 /$ insitu.6493

ISSN : 1630-7305

\section{Éditeur}

Ministère de la culture

\section{Référence électronique}

Marc Saboya, «Le campus et le C.H.U de Bordeaux 1959-2005», In Situ [En ligne], 17 | 2011, mis en ligne le 06 avril 2012, consulté le 19 avril 2019. URL : http://journals.openedition.org/insitu/6493 DOI : 10.4000/insitu.6493

Ce document a été généré automatiquement le 19 avril 2019

\section{(c) (i) $\odot$}

In Situ Revues des patrimoines est mis à disposition selon les termes de la licence Creative Commons Attribution - Pas d'Utilisation Commerciale - Pas de Modification 4.0 International. 


\title{
Le campus et le C.H.U de Bordeaux 1959-2005
}

\author{
Marc Saboya
}

\section{Première partie : Le campus et le CHU : 1959-1974}

Le campus, style « sévère »

1 Le 23 juillet 1963, le plan masse du complexe universitaire prolongeant les premières réalisations de René-André Coulon est approuvé par le Conseil général des bâtiments de France ${ }^{1}$. L'extension, programmée dès le départ mais impulsée par le recteur Babin, s'étire sur près de 250 ha. Le campus de Talence, Pessac et Gradignan est désormais un des plus grands d'Europe. Le plan, confié à l'architecte parisien Louis Sainsaulieu, spécialiste des constructions scolaires, présente une répartition des principales unités le long d'un axe est-ouest matérialisé par l'avenue des Facultés. De part et d'autre de cette voie Sainsaulieu dispose des bâtiments dont l'emprise au sol ne représente que $10 \%$ du terrain disponible, le reste étant occupé par des terrains de sport et des espaces verts ${ }^{2}$. Trois grands ensembles se détachent le long de cet axe boisé : l'extension de la faculté des sciences, la faculté de droit puis la faculté des lettres. Villages et restaurants universitaires, complexes sportifs, instituts et écoles spécialisées viennent s'inscrire autour de ces pôles.

2 (fig. $\left.\mathbf{n}^{\circ} \mathbf{1}\right)$ 


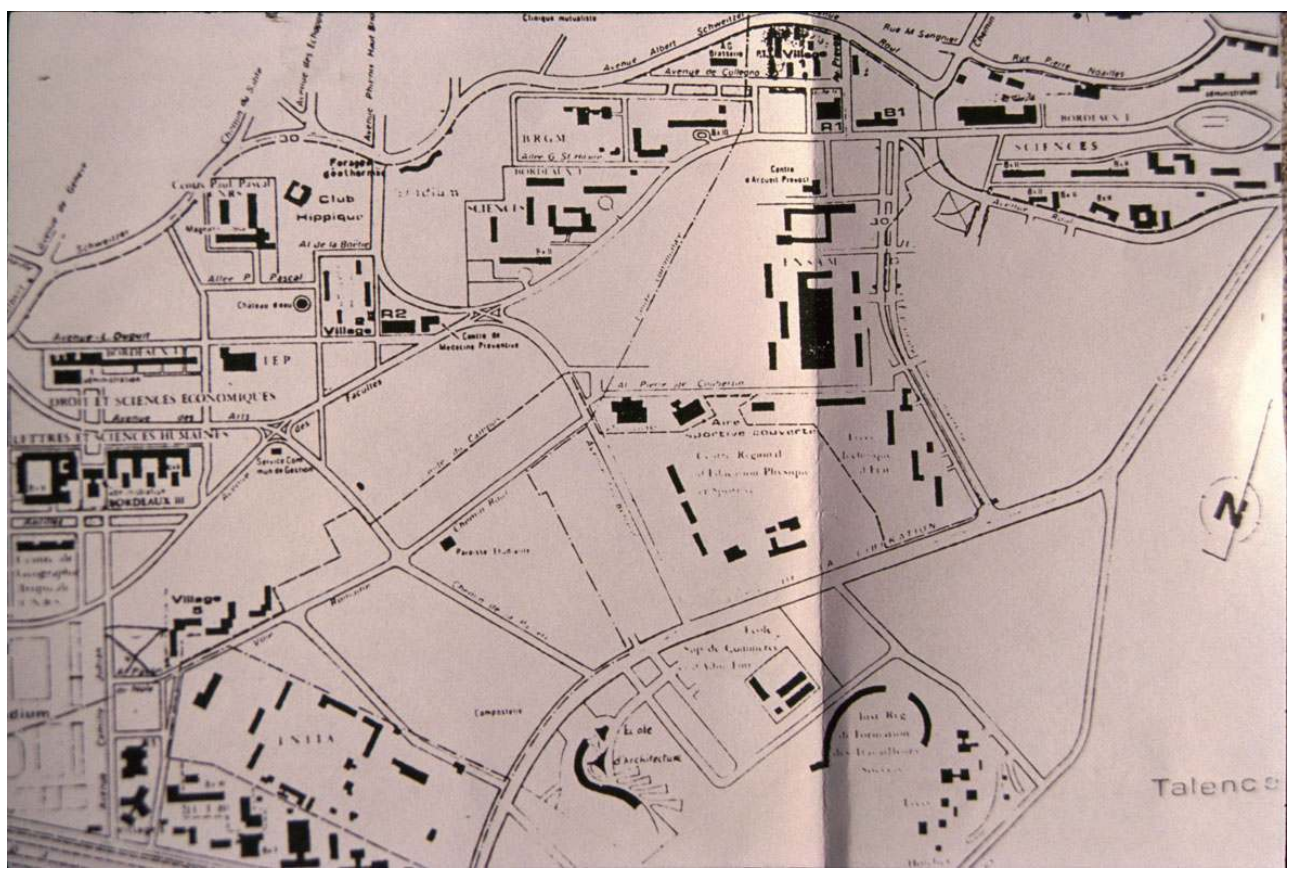

Détail du plan de masse du campus, Louis Sainsaulieu, 1963 : à gauche, face à face, les facultés de droit et de lettres.

(c) Marc Saboya.

3 Au-delà de l'élégant pont des Universités (Francisque Perrier, 1971) s'étirent les nouveaux bâtiments de la faculté des sciences réalisés, pour la troisième tranche, entre 1966 et 1967 par Jean-Louis Ludinart (René-André Coulon, architecte en chef). Le parti reprend les formes, couleurs et matériaux du programme précédent dans une composition aérée et agrémentée de trois sculptures de Jean Bertoux. Au sud, Jacques Carlu et André Comte achèvent en 1966 le lycée technique d'état et l'importante École nationale supérieure des Arts et Métiers, un ensemble de quatre barres basses enserrant une vaste cour rectangulaire sur laquelle se greffe une aile occupée par l'amphithéâtre et les laboratoires tandis qu'à l'autre extrémité une haute tour est occupée par les logements de fonction ${ }^{3}$. La disposition des bâtiments, leur échelle, le rationalisme du parti architectural renvoient aux prestigieux modèles américains tel le centre universitaire de Harvard (Cambridge, Massachusetts) par Walter Gropius en 1950. L'entrée de l'école est signalée par une haute sculpture d'Alicia Penalba, sculpteur argentin élève de Zadkine. Partiellement enterrée, la bibliothèque des sciences (1963-1964, L. Sainsaulieu et P. Daurel), longue cage de verre rythmée par les blocs de béton des rayonnages qui semblent suspendus aux parois vitrées, joue sur la transparence et évoquerait, bien sûr, l'architecture de Mies van der Rohe si certains éléments, tels les fermes triangulaires et les plafonds inclinés, n'introduisaient des effets plastiques étrangers à l'art du maître mais issus des dernières expériences de Le Corbusier et de Jean Prouvé. À l'arrière, contrastant avec la limpidité de la salle de lecture, la tour des magasins élève ses murs de béton rythmés uniquement de fentes verticales. La même lisibilité, les mêmes effets sont recherchés par les deux architectes à la bibliothèque juridique et littéraire (1967-1968) qui ferme l'extrémité ouest du complexe universitaire mais s'élève sur quatre niveaux. À l'intérieur, le principe d'une ossature métallique en poteaux-poutres permet de libérer de vastes plateaux que l'on peut aménager à la demande. À l'instar du bâtiment précédent, les façades sont revêtues 
de mosaïques de pâte de verre blanche mais ici un portique forme un auvent sous lequel se glisse l'entrée monumentale de l'édifice qui souligne l'axe de la composition s'achevant, à l'arrière, par la grande tour des magasins.

\section{(fig. $\left.n^{\circ} 2\right)$}

Figure 2

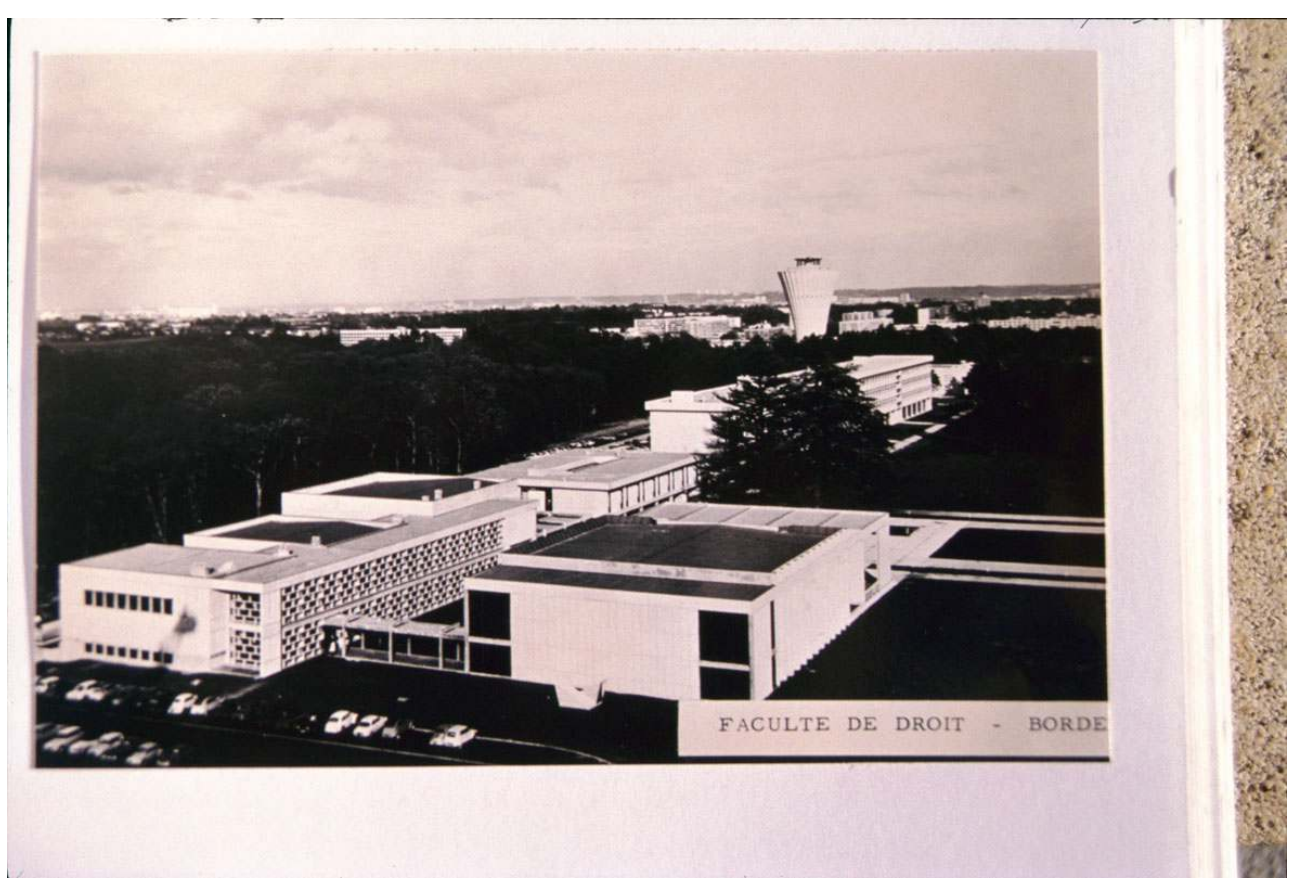

Louis Sainsaulieu et Pierre Mathieu : faculté de droit et de sciences économiques, Pessac, 1965-1967. (c) Marc Saboya.

Près du château d'eau, haut calice de béton au cœur du domaine universitaire (1962, Pierre Mathieu), l'Institut de magnéto-chimie-C.N.R.S. (1964-1965, P. Mathieu, A. Bersagol, R. Tagini) déploie un long mur-rideau de verre coloré. Plus à l'ouest, l'Institut d'études politiques forme la transition vers le secteur droit-lettres (1967, P. Mathieu, P. Daurel, C. Bouey). Édifice autonome, il se présente comme un quadrilatère bas avec des extensions greffées sur deux angles pour des amphithéâtres et la recherche. Le patio central est aménagé en jardin visible de l'extérieur grâce aux parois de verre du hall d'entrée.

L'ensemble droit-lettres a fait l'objet d'un véritable projet urbain et architectural dont la cohérence a été partiellement détruite par l'ajout postérieur de nombreux bâtiments que le programme n'avait pas prévus. L. Sainsaulieu et P. Mathieu disposent les deux facultés en un intéressant face à face de part et d'autre de l'esplanade Michel-Montaigne traversée par l'avenue des Arts. Lettres au sud, droit au nord s'étirent en deux longs bâtiments parallèles. À la cour d'honneur de la faculté des lettres, fermée par le rideau de verre de l'administration et ornée d'une composition de Marc-Antoine Loutre ${ }^{4}$, répond le vaste parvis formé par l'avancée du grand amphithéâtre de droit, Aula Magna, marqué par la fontaine pétrifiée de Yasuo Mizui ${ }^{5}$. Construite de 1965 à 1967, la faculté de droit et des sciences économiques s'organise en trois parties distinctes: l'administration, les amphithéâtres et l'aile des salles de cours et de travaux pratiques. Les amphithéâtres de 500 à 200 places sont groupés à l'ouest derrière un long claustra d'alvéoles de béton 
faisant office de brise-soleil et protégeant un mur rideau de verre. La façade nord affirme les volumes bruts des salles dont les murs aveugles sont revêtus de plaques de gravillons lavés. L'Aula Magna de mille places est traité séparément, en retour d'équerre, mais relié à cet ensemble par une galerie couverte. Son importance, sa situation privilégiée appelaient un traitement monumental. Pierre Mathieu le conçoit comme un temple auquel on accède par plusieurs emmarchements qui l'élèvent, tel un palais de justice moderne, au-dessus des passions terrestres. Un profond pronaos au plafond à caissons est porté par des voiles de béton qui évoquent la colonnade antique. L'administration est un bâtiment bas dont les baies étroites sont scandées par des blocs qui assurent le contreventement. La salle du conseil, à l'étage, est décorée d'une grande tapisserie en laine de Michel Seuphor, Cos (1968). Au-delà s'étire la longue aile de l'enseignement unifiée par la scansion des poteaux de béton et les plaques de gravillons lavés. (fig. $\mathbf{n}^{\circ} \mathbf{3}$ )

Figure 3

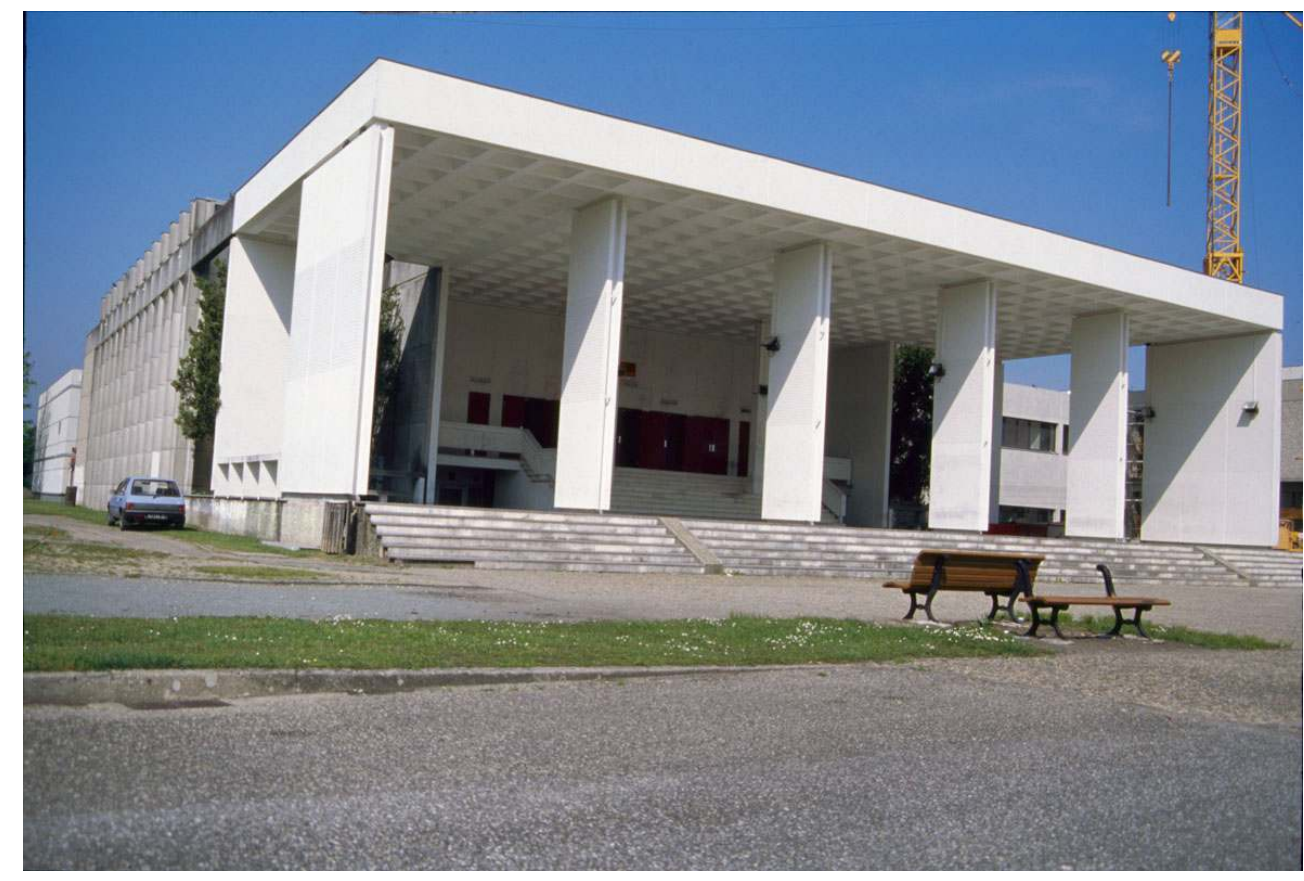

Pierre Mathieu, Claude Bouey, Paul Daurel : Aula Magna, faculté de droit et de sciences économiques, Pessac, 1965-1967.

(C) Marc Saboya.

7 En face s'organisent les bâtiments de la faculté des lettres et sciences humaines (1965-1970, P. Mathieu, P. Daurel, R. Tagini) selon un parti plus dynamique d'ailes greffées en retour d'équerre sur un long bâtiment et dessinant ainsi trois vastes patios sur lesquels donnent, au sud, les parois vitrées des amphithéâtres reliés par une galerie ouverte ${ }^{6}$. En retrait sur l'esplanade, l'administration déploie ses rideaux de verre rythmés par l'ossature de béton. La salle des actes, à l'étage, est ornée d'une tapisserie de Vieira da Silva, La Ville (1968). À l'arrière, le grand amphithéâtre de 700 places offre une originale ordonnance de piliers de béton au profil triangulaire en pyramide inversée portant un avant-toit incliné vers l'intérieur de la salle. Ce parti plastique, fréquent dans l'architecture des années soixante et rappelant certaines formules d'Oscar Niemeyer, conjugué à une audace technique relative, justifie la place accordée à cette œuvre dans les premiers ouvrages consacrés à l'histoire de l'architecture contemporaine française ${ }^{7}$. À 
l'ouest, un dernier corps de bâtiment entoure une grande cour carrée plantée de nombreux arbres. Pour adoucir l'austérité du parti orthogonal de l'ensemble des élévations, P. Mathieu a travaillé la peau de ses façades par des inclusions de cailloux lavés, par un béton brut de décoffrage dont les banches dessinent des rayures verticales ou horizontales (mur aveugle de l'amphi 700), par des panneaux lisses ou par de longues frises de motifs géométriques moulés. Ces recherches plastiques produisent des contrastes de relief et de texture qui permettent de différencier les façades et les diverses fonctions des bâtiments. Elles caractérisent aussi les bâtiments de recherche installés près de la faculté des lettres. Le centre de géographie tropicale (1967, P. Mathieu) présente un corps central d'articulation entièrement vitré ; à la maison des sciences de l'homme (1972-1976, P. Daurel) les pleins de travée sombres alternent avec les plaques de béton brut. L'entrée est soulignée par un auvent de béton brut de décoffrage avançant dans le vide comme une vague, un traitement plastique qui évoque les portiques et les toitures de Le Corbusier à Marseille et à Chandigarh. (fig. $\left.\mathbf{n}^{\circ} 4\right)\left(\right.$ (fig. $\left.\mathbf{n}^{\circ} 5\right)\left(\right.$ fig. $\left.^{\circ} \mathbf{6}^{6}\right)$

Figure 4

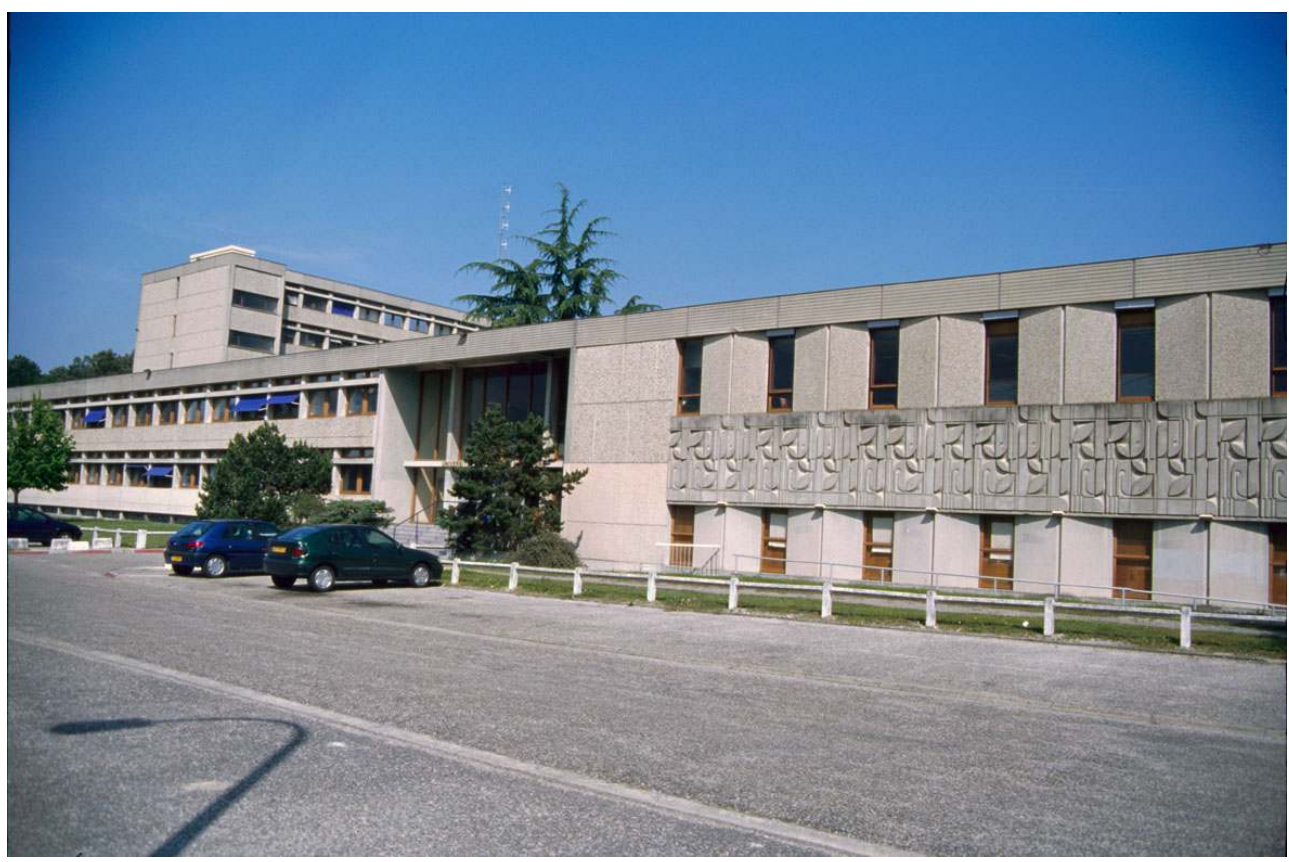

Pierre Mathieu, Paul Daurel, Tagini : faculté des lettres, façade sud, Pessac, 1968.

(C) Marc Saboya. 
Figure 5

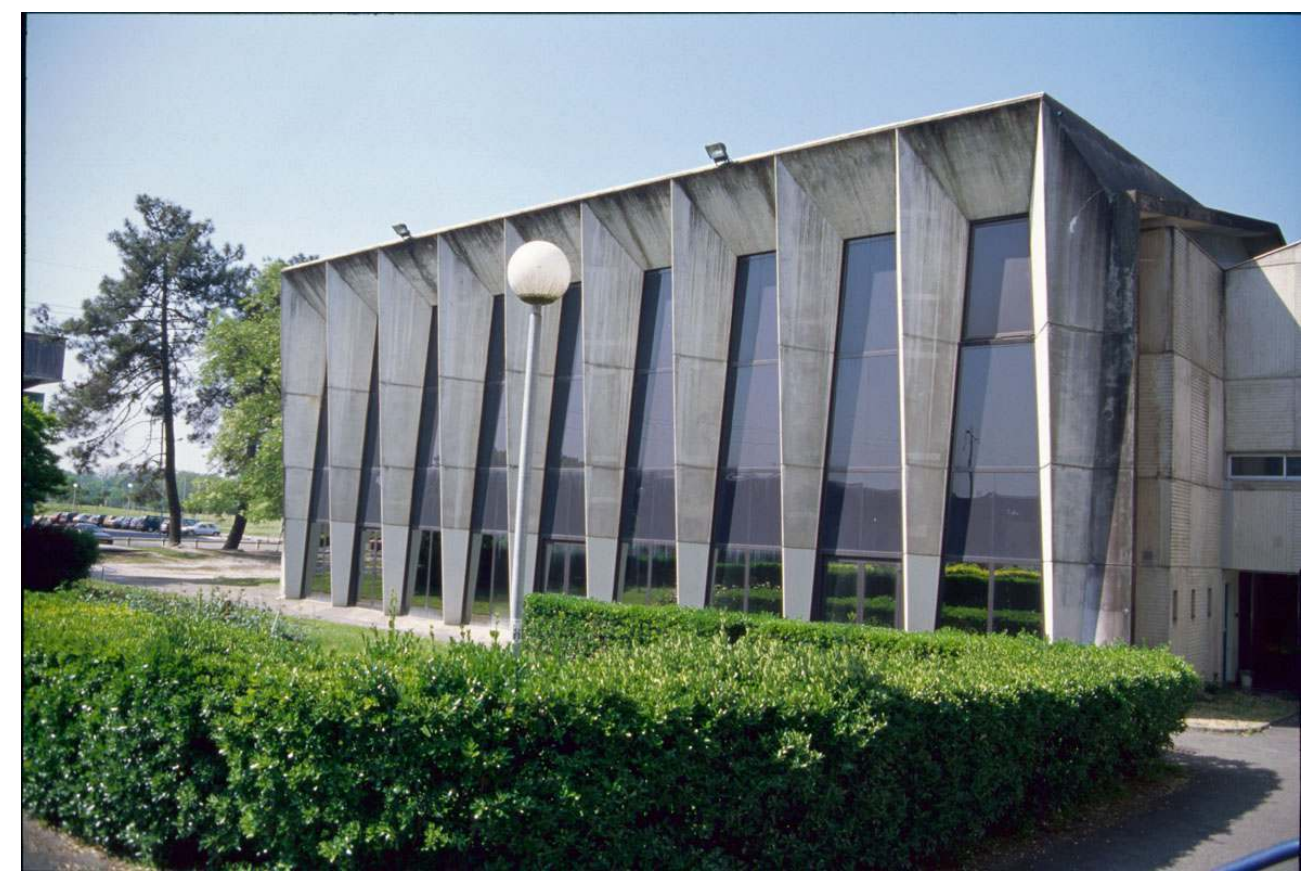

Pierre Mathieu, Paul Daurel, Tagini : faculté des lettres, amphi 700, Pessac, 1968.

(c) Marc Saboya.

Figure 6

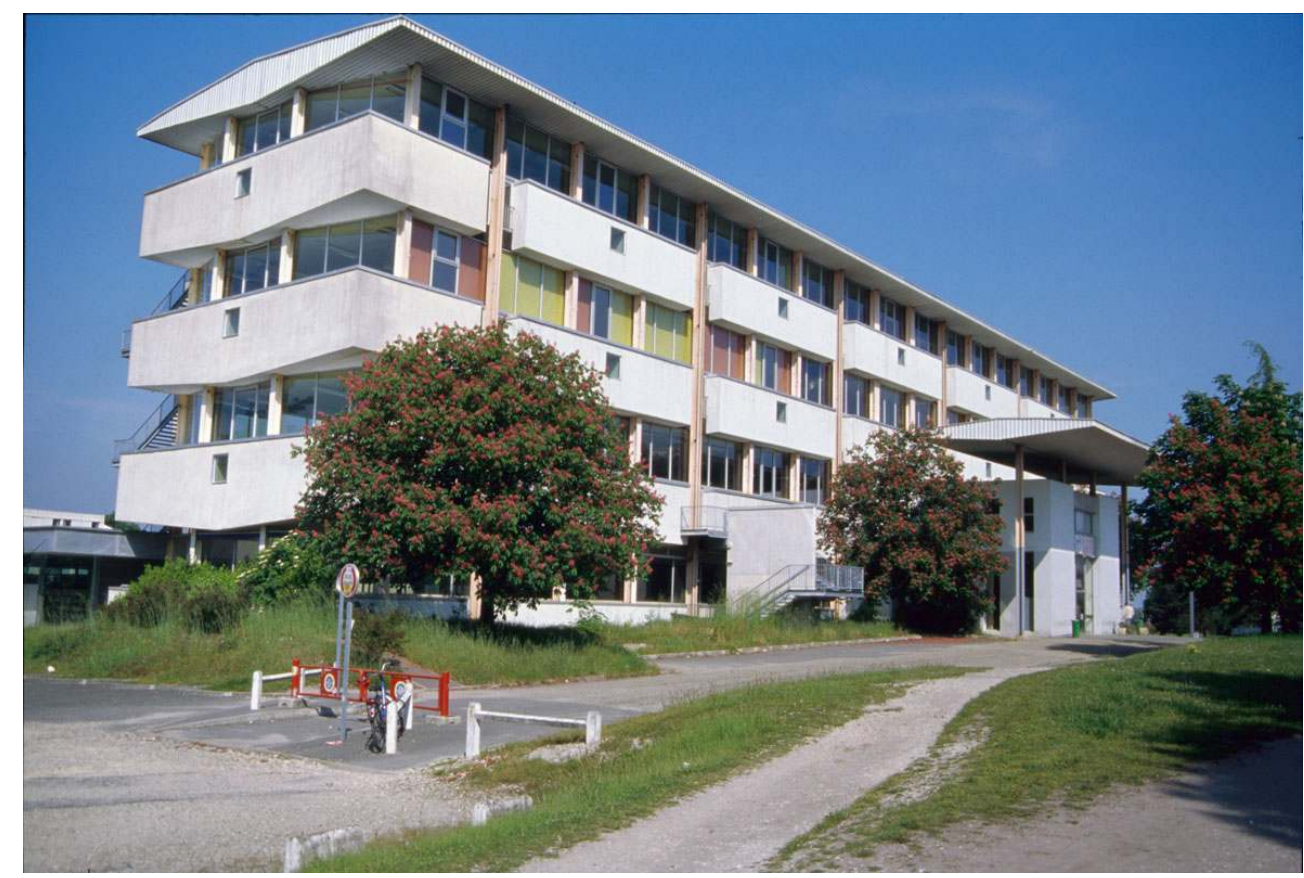

Louis Sainsaulieu, Daurel : bibliothèque Droit-Lettres, Pessac, 1968.

(c) Marc Saboya. 


\section{L'École d'architecture, de la place Sainte-Croix (Bordeaux) au domaine de Raba (Talence) : crise (1968) et renouveau}

Le décret du 6 décembre 1968 institutionnalise la séparation entre l'École des beaux-arts et l'École d'architecture en créant des Unités pédagogiques (U.P.) parisiennes et provinciales remplaçant les anciennes écoles. En attendant l'installation de l'U. P. de Bordeaux dans de nouveaux bâtiments à Talence, les étudiants sont logés dans des baraquements préfabriqués proches du quai Sainte-Croix libérés par les Anciens Combattants. Grâce à l'appui de J. Chaban-Delmas et à l'intervention de Ferret, l'École de Bordeaux est alors la seule qui fonctionne avec deux directeurs: un directeur administratif, Yves Lormant, très souvent contesté par les professeurs et les élèves, et un directeur des études, C. Ferret lui-même qui confirme ainsi son autorité et continue à imposer un enseignement classique de grande qualité mais fermé aux matières nouvelles : "Il montrait une architecture occidentale et dans cette architecture occidentale, une architecture européenne et dans cette architecture européenne une architecture d'architecte, dans cette architecture d'architecte une architecture monumentale» dira plus tard Jean-Paul Loubes. Sous la haute direction de Claude Ferret survivent, dans des conditions précaires, les trois ateliers de Serge Bottarelli, Jean Sérieis et Yves Salier dont les méthodes pédagogiques sont diversement appréciées ${ }^{8}$. L'École est désormais le lieu d'un débat riche et permanent qui s'exprime au sein du Conseil de gestion, émanation des revendications des étudiants et des enseignants. Les fils d'architectes s'opposent parfois vigoureusement à ceux qui réclament la fin du mandarinat. Des revues internes témoignent avec humour de la virulence de certains de ces échanges et c'est un étudiant bordelais, Christian Maudet, signataire des Propositions de Tarbes, qui est choisi comme représentant national de la coordination des étudiants en architecture.

(fig. $n^{\circ} 7$ ) 
Figure 7

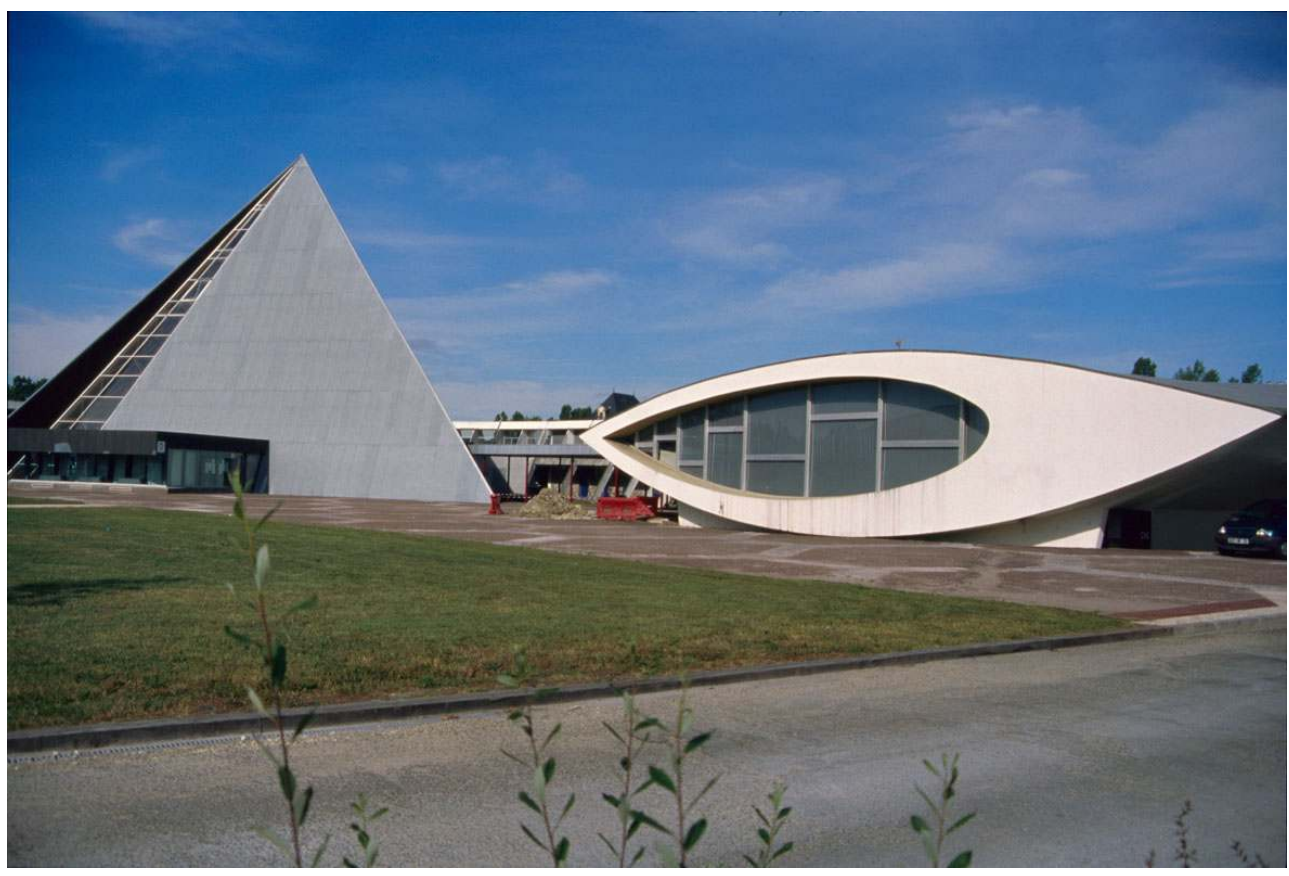

Claude Ferret : pyramide et amphithéâtre, École d'architecture, Talence, 1970-1973.

(c) Marc Saboya.

Si Pierre Lajus apporte, quelque temps avant de se retirer en 1970, les notions d'urbanisme réclamées par les étudiants, ceux-ci réitèrent leurs revendications pour un enseignement de l'ethnologie, de la psychologie et de la sociologie dont C. Ferret aurait dit qu'elle "est à l'architecture ce que la martingale est à l'imperméable »". Edmond Lay, un moment sollicité, ne peut rejoindre l'équipe. Claude Ferret lui préfère en effet Jean Sérieis (méthodologie du projet) qui devient un enseignant très apprécié tandis que Pierre Anus propose sa connaissance de l'Égypte, de l'architecture de terre et de l'écologie. Pourtant l'institution ne semble pas accepter une contestation qui passe par le rejet du professionnalisme traditionnel, se penche sur le caractère politique et social de l'architecture et de l'urbanisme et tente de se rapprocher des sciences humaines. De nombreux étudiants quittent alors une école qu'ils jugent trop traditionnell ${ }^{10}$ ou subissent un enseignement qu'ils considèrent comme "une grande entreprise de démoralisation ${ }^{11}$; d'autres - tels Piou Lacoste et Jean-Paul Loubes - s'intéressent aux expérimentations sur l'énergie solaire, aux travaux des charpentiers américains, aux structures complexes (polyèdres, structures géodésiques), à l'architecture sans architectes: "ce qui nous intéressait au début des années soixante-dix c'était comment un architecte devient un homme normal et non un démiurge $»^{12}$. Pour les plus jeunes, ils étaient "une bouffée d'oxygène» diront d'eux Anne Lacaton et Jean-Philippe Vassal qui entrent alors à l'École (1974-1975), "ils ne parlaient pas comme les autres, ils ne parlaient pas de façades, ils parlaient d'autre chose ${ }^{13}$ ».

11 Dans l'attente d'une pluridisciplinarité très demandée par les étudiants qui veulent un enseignement proche de celui donné à l'université, une pratique à l'écoute de la vie quotidienne et qui souhaitent que l'architecture soit une activité collective (un diplôme est soutenu en 1974 par Piou Lacoste et Jean-Paul Loubes sur un programme d'autoconstruction et d'autogestion dans les Landes) ${ }^{14}$, les débats vont se cristalliser 
autour de la construction de l'école, le ministère ayant décidé la réalisation de nouveaux locaux pour les U.P. de province. Trois projets associant étudiants et enseignants sont en présence : une proposition utopique formée de petites sphères abritant les activités (J. Heim), une composition centrée sur un vaste forum couvert d'une coque de cuivre, sorte de lieu de débat et d'enseignement autour duquel se greffent différentes salles (Ch. Maudet et L. Cazalis, J. Gourvelec, initiateurs du projet et, pour la plupart, signataires des Propositions de Tarbes) et un projet éclaté présenté par les élèves du "patron " parmi lesquels B. Trinqué. Dès les premières esquisses, Ferret choisit le troisième projet. Malgré les réclamations et interventions des représentants de la deuxième proposition, le ministère entérine le choix de Claude Ferret. Sans parler véritablement d'œuvre collective il faut admettre que le projet Ferret est un travail de concertation et d'échanges au sein de l'atelier du maître ${ }^{15}$.

C'est peut-être la forme dynamique de l'amphithéâtre de l'École supérieure de commerce voisine (1968, F. Perrier) qui inspire Claude Ferret lorsqu'il construit l'École d'architecture toute proche (1970-1973). Le plan de l'ensemble est assez complexe: un point d'interrogation inversé pour certains, une feuille d'acanthe rappelant le chapiteau corinthien pour d'autres. Le formalisme de ce plan renvoyant à la tradition classique de l'architecture parlante (voir C.N. Ledoux) est sensé traduire la fluidité des déplacements au sein de l'école, le traitement des circulations étant une des qualités des projets de C. Ferret à l'échelle d'une ville (Royan) comme à celle d'un bâtiment (caserne des pompiers rive droite). Le long de cette colonne vertébrale ondulante occupée par les salles de cours, les bureaux et doublée par une galerie ouverte, l'architecte accroche cinq grands ateliers répartis en éventails, un espace de rencontre et un amphithéâtre. Formellement, chaque espace est différencié par un traitement particulier. Les volumes des ateliers s'évasent progressivement et les toits suivent, par une courbe accentuée, ce mouvement ascendant. Le point de rencontre est une haute pyramide et l'amphithéâtre est traité comme un œil monumental qui peut évoquer une fois encore C. N. Ledoux mais s'inspire surtout de l'amphithéâtre de Belo Horizonte (Brésil) construit par O. Niemeyer en 1956. Chaque bâtiment revendique une autonomie formelle comme autant de sculptures, d'objets architecturaux dispersés le long d'une ligne ondulante. Le lyrisme à la brésilienne, l'exubérance formelle qui caractérisaient certaines réalisations royannaises s'affirment ici dans une des dernières œuvres de l'architecte directeur de l'école. Mais le parti est trop affirmé pour s'adapter à la nouvelle politique des écoles régionales et satisfaire, après mai 1968, les exigences des étudiants. Selon Gilles Ragot, la pyramide ne fonctionne pas comme un lieu de rencontre et de convivialité mais comme un signal à l'échelle urbaine ${ }^{16}$. L'éclatement architectural, accentué par l'absence de couloirs, favorise le fractionnement des disciplines et renforce l'enseignement du "patron » alors que, paradoxalement, la tendance officielle, née de 1968, est à la pluridisciplinarité. CEuvre inadaptée et atypique où «le formalisme souligne jusqu'à l'évidence le découpage fonctionnaliste du plan et de sa distribution ${ }^{17}$ " l'école de Bordeaux est un peu à l'image de la forte personnalité de Claude Ferret, maître autoritaire formé à l'École des beaux-arts de Paris qui, à l'heure où se dessine un renouveau pédagogique, affirme la position de l'atelier autonome et veut conserver l'architecture comme une science indépendante des autres disciplines. "Il nous faisait dessiner l'architecture moderne comme un objet » déclara Pierre Lajus qui continuait: " il y avait ceux qui étaient dans le parti et ceux qui n'étaient pas dans le parti du patron ». Ce point de vue, que partage une partie des jeunes contestataires entrés à l'École en 1968 et 1969, doit être nuancé. Nombreux sont ceux qui, formés par 
Ferret dans les années soixante - tel Serge Bottarelli, Brigitte Gonfreville - ou soixantedix - tel Patick Hernandez - reconnaissent la qualité de l'enseignement du maître ${ }^{18}$.

\section{Le CHU}

Depuis la loi Debré (1958), les facultés de médecine doivent faire partie d'un complexe hospitalier. Cette association en un grand centre scientifique est déjà mise en œuvre aux États-Unis où se développe le principe de l'hôpital-bloc qui s'oppose au programme pavillonnaire. La faculté de médecine de Bordeaux (Bordeaux II) va donc s'établir au voisinage du complexe hospitalier de Carreire (Pellegrin). Pierre Mathieu contrôle et coordonne les opérations qui débutent en 1967 avec l'approbation du plan masse. Dans un environnement vert, le plan s'ordonne sur un tracé orthogonal donné par les circulations, les galeries de liaison et les axes des bâtiments, le parti architectural étant guidé, selon Gilles Ragot, par le respect des trames préétablies et le recours à la préfabrication lourde ${ }^{19}$ . La première tranche, au sud, est réalisée par Paul Daurel, André Conte et Jean-Jacques Prévot (1970-1978). Il s'agit d'un grand hall d'accueil, du bâtiment de l'administration, de six amphithéâtres et des bâtiments d'enseignement dirigé et de travaux pratiques. Ces derniers sont construits avec une ossature de béton armé formant des portiques transversaux disposés tous les trois mètres. Cette trame répétitive permet de prévoir des éléments de façade préfabriqués revêtus d'un parement de cailloux lavés. Les amphithéâtres adoptent une structure particulière de poteaux extérieurs qui portent les toitures et libèrent le maximum d'espace intérieur. Sur les murs en parpaings des plaques de béton dessinent un jeu de pointes de diamant. À l'instar des halls des facultés de lettres et droit, le grand hall de Bordeaux II est entièrement vitré. Le grand amphithéâtre de 800 places construit en contrebas de la route est légèrement antérieur aux débuts des travaux du complexe universitaire. Projeté en 1968, il offre un contour hexagonal original dentelé par les poteaux pare-soleil qui l'entourent. La seconde tranche, au nord, commencée en 1972, comprend les laboratoires de recherche. C'est une longue construction suivant un plan en peigne, intimement liée à l'imposant tripode, hôpitalbloc de 13 étages, 2300 lits et 3 ailes qui donne son identité au complexe de Pellegrin (1974-1978, J. Sérieis, P. Mathieu, J. Tournier-Ardilouze $)^{20}$. La bibliothèque enfin (1971-1974, P. Daurel, A. Conte, J.-J. Prévot) est un bâtiment compact où les fenêtres, rares sur les façades est et ouest, alternent avec les saillies vigoureuses et brutalistes de coques de béton brut de décoffrage. 


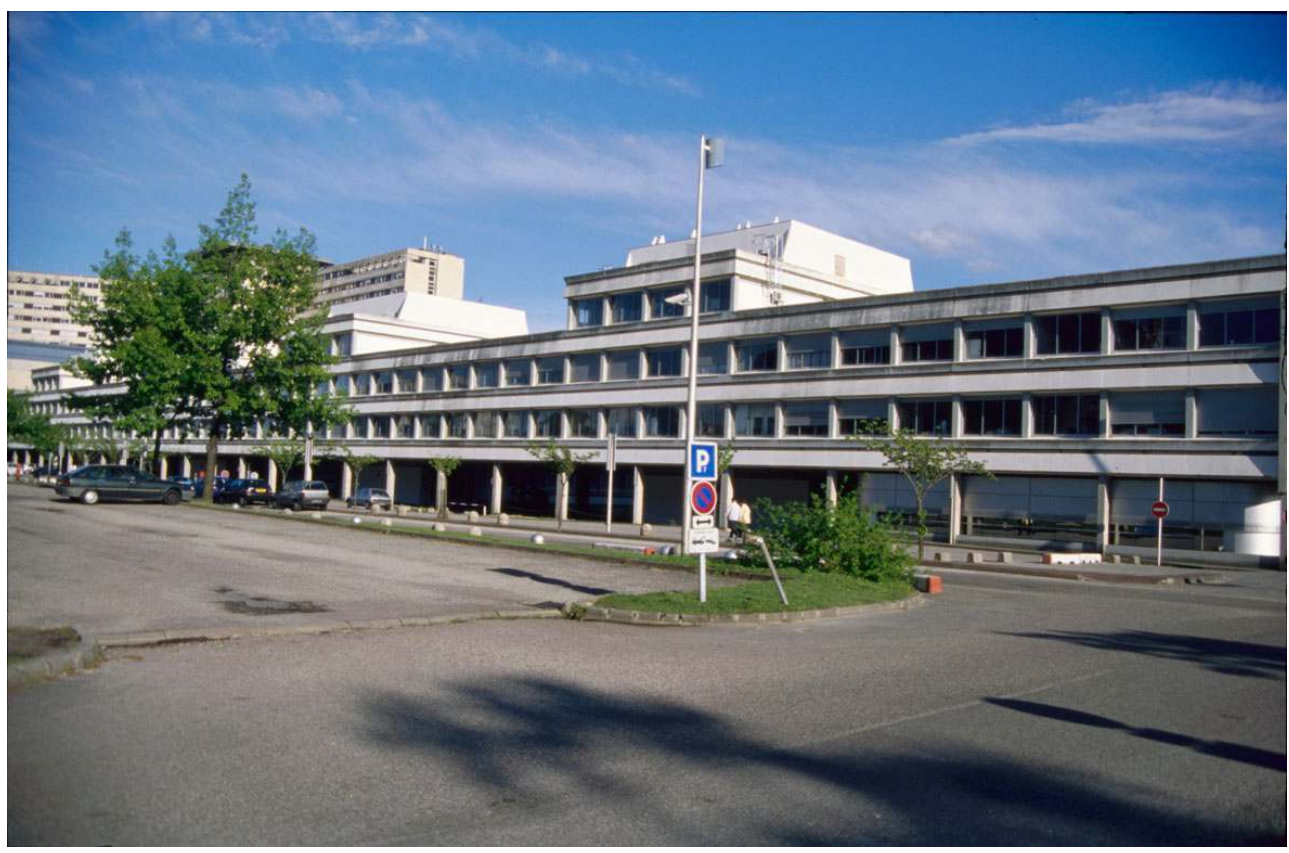

Sérieis, Mathieu, Guérin : CHU-Pellegrin, Bordeaux, 1973.

(c) Marc Saboya.

Figure 9

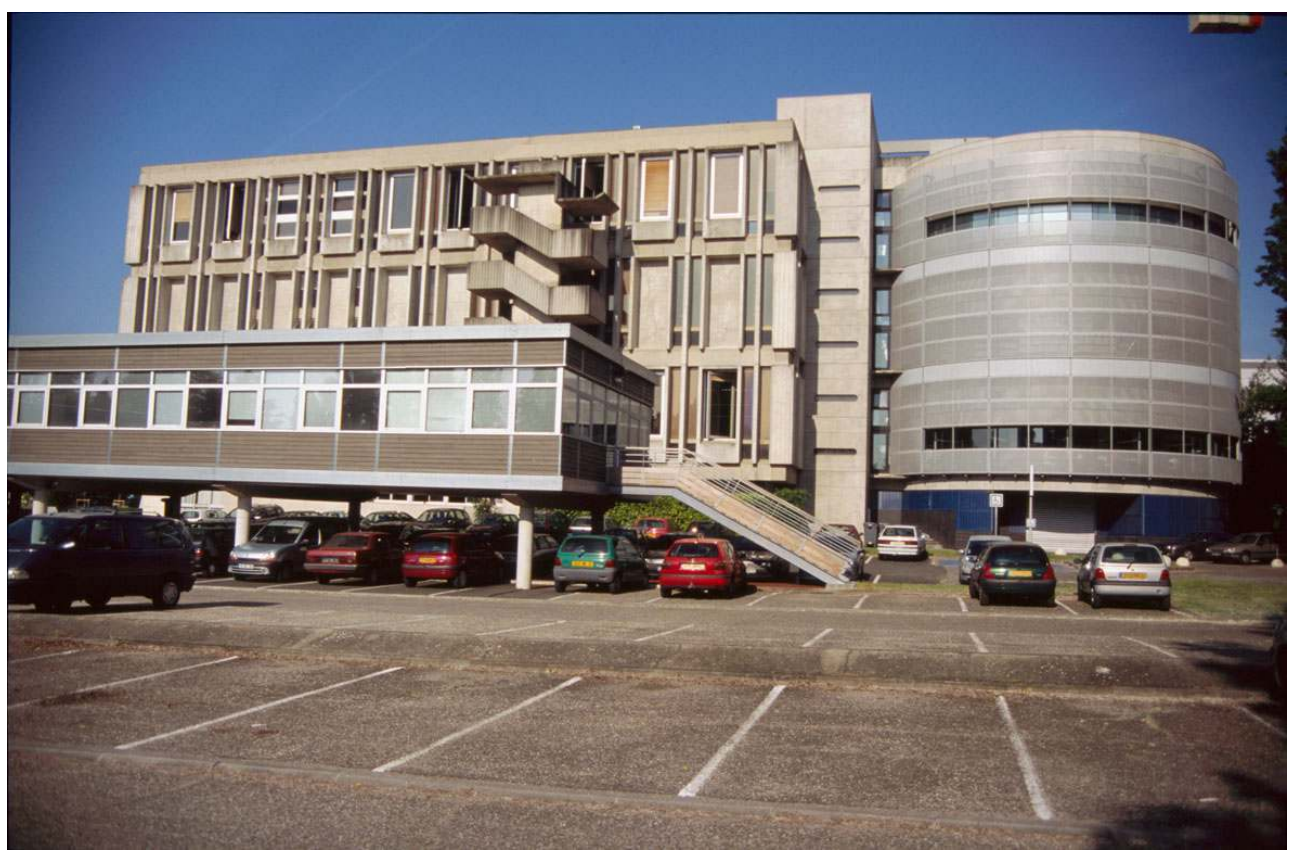

Daurel, Conte, Prévost : bibliothèque de la faculté de médecine et de pharmacie, CHU-Pellegrin, Bordeaux, 1971-1974. Extension (la rotonde) 1993, Bouey, Ragueneau, Roux.

(c) Marc Saboya.

15 Au sein de ce fonctionnalisme rigoureux l'Institut de formation aux carrières de la santé (1973, Sérieis, Guérin, Mathieu) à l'extrémité ouest du domaine est totalement atypique. Grand parallélépipède bas allongé, le bâtiment déborde de son socle et semble ainsi 
flotter sur les poteaux de structure qui évoquent des pilotis. Cette référence corbuséenne est confortée par l'immense toit plat qui couvre l'ensemble et par la quarantaine de paresoleil qui rythment la façade ouest et protègent ainsi l'amphithéâtre et la bibliothèque. Le décor de ces pare-soleil est très original : chaque panneau est recouvert d'un motif de mosaïque qui devient un élément d'une vaste composition colorée lorsqu'on aborde l'édifice de biais. Selon l'angle de vision, la longue frise forme des vagues à dominante orange et beige (de droite à gauche) ou jaune et grise (de gauche à droite). Grand geste " pompidolien » évoquant les compositions cinétiques contemporaines de Yaacov Agam, ce décor est une des rares réussites bordelaises dans la tentative des années soixante-dix d'intégrer les arts plastiques à l'architecture ${ }^{21}$.

\section{(fig. $\left.n^{\circ} 10\right)$}

\section{Figure 10}

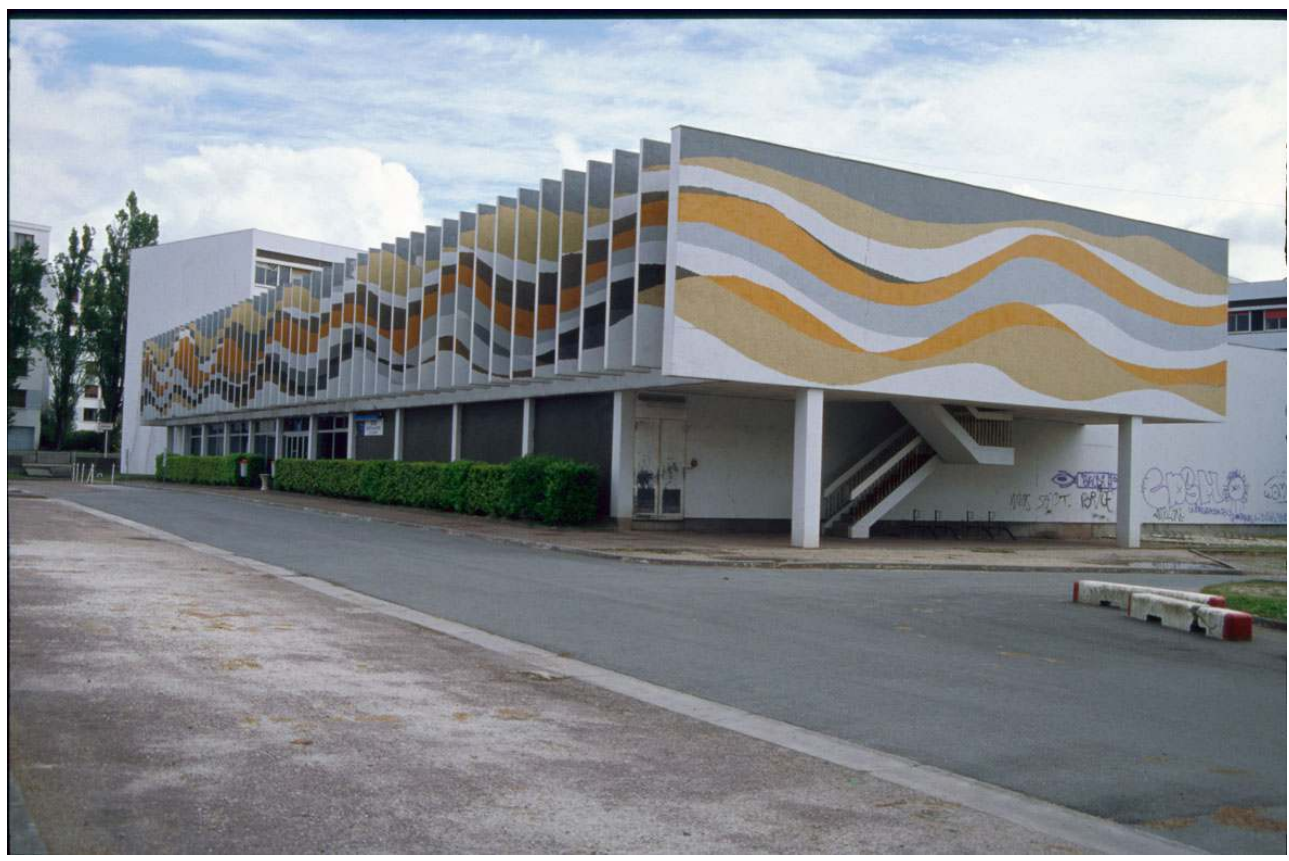

Sérieis, Mathieu et Guérin : institut de formation des carrières de la santé, en marge du CHU, 1973.

(c) Marc Saboya.

En dépit des nombreuses modifications qui ont considérablement altéré le parti des bâtiments, les ouvrages qui forment le noyau originel de l'immense domaine universitaire sont d'excellents témoignages du fonctionnalisme des années soixante et traduisent une intelligence des matériaux et des espaces, un respect des besoins de l'utilisateur qui sont les marques d'une architecture de grande qualité. S'ils n'ouvrent pas des voies nouvelles à l'art de bâtir, ils prouvent que "l'architecture moderne a atteint sa maturité. Il faut ce fond de certitude pour permettre à d'autres une recherche plus prospective " déclare Jean Fayeton dans un numéro de 1964 de L'Architecture d'aujourd'hui faisant un bilan des réalisations françaises ${ }^{22}$. 


\section{Deuxième partie : Les pôles universitaires 1996-2005: fin du style " sévère »}

En 1990, face à l'augmentation considérable du nombre d'étudiants, le plan Université 2000 s'accorde cinq ans pour construire 1,5 millions de mètres carrés sur les campus et resserrer les liens entre les villes et leurs universités ${ }^{23}$. Des mesures incitatives (financement, maîtrise d'ouvrage) suscitent dans la plupart des centres universitaires de nombreux projets qui renouvellent la typologie des bâtiments. Sur le domaine universitaire de Bordeaux-Talence-Pessac ainsi qu'au C.H.U. de Pellegrin - les nouvelles constructions vont considérablement transformer la monumentalité classique et sévère d'une architecture de barres datant de la fin des années soixante. La confrontation avec les anciens bâtiments - qui eux-mêmes subissent des modifications altérant les partis d'origine (patios construits, amphithéâtres morcelés en salles de cours, fermeture de galeries ouvertes) - donne aux architectes l'occasion d'expérimenter des solutions surprenantes comme autant de gestes personnalisés tranchant vigoureusement dans le rationalisme ambiant. Les œuvres que nous avons retenues sont toutes profondément marquées par la personnalité de leurs créateurs et sont les authentiques produits de la culture d'une équipe, d'une agence. Aucun édifice n'est banal, ordinaire; tous sont marqués par la volonté de secouer - ou de ré-interpréter - l'héritage du Mouvement moderne sans tomber dans les facilités du postmodernisme. Grâce à ces œuvres, le campus a retrouvé de la couleur, de la lumière, une symbolique, le plaisir du mouvement et des formes plastiques ainsi qu'une dimension ludique et onirique qui pourrait rajeunir voire ébranler l'austérité de l'acte pédagogique.

19 (fig. $\left.\mathbf{n}^{\circ} \mathbf{1 1}\right)$

Figure 11

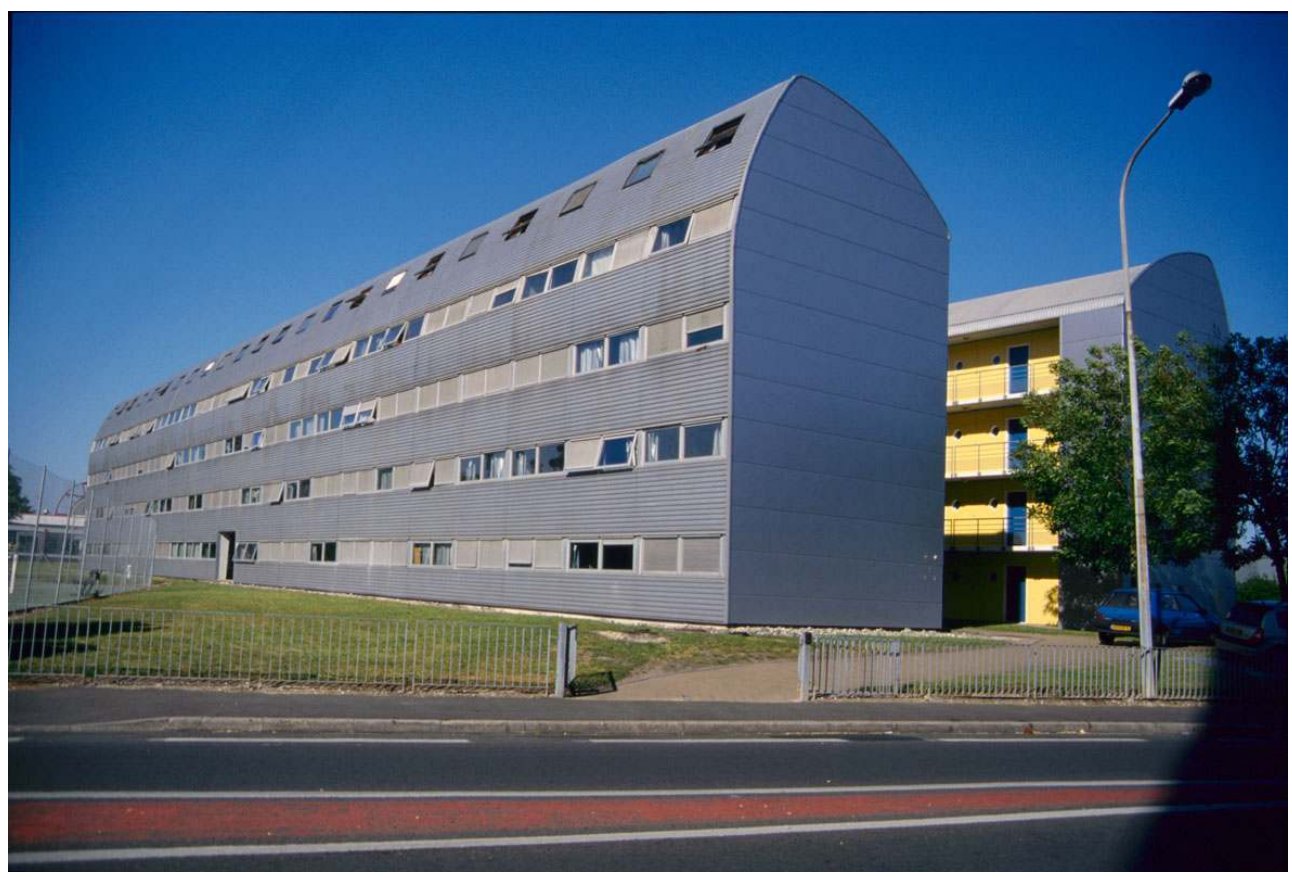

Alain Loisier, Jean de Giacinto : résidence « Campus », Talence, 1991.

(C) Marc Saboya. 
Avec l'augmentation du nombre d'étudiants, l'apparition de nouveaux centres d'intérêt dans l'enseignement et la recherche, le déplacement vers la périphérie des grandes écoles et des instituts, l'éclatement de la structure universitaire traditionnelle multipliant les structures satellites de la maison-mère (maison des pays ibériques, maison de l'archéologie, maison des arts etc.) vont favoriser la prolifération de constructions nouvelles originales. Edmond Lay à l'I.R.T.S. et Claude Ferret avec l'École d'architecture avaient, au début des années soixante- dix, ouvert la voie d'une architecture où s'exprimait une recherche formelle évidente en conflit avec la dominante rationaliste de l'environnement. En 1985, Paul Daurel bâtit une Maison des pays ibériques de type méditerranéen avec patio, arcades et toit de tuile en pente douce. En 1991, sur le cours de la Libération, Alain Loisier et Jean de Giacinto livrent la résidence Campus construite en six mois à la suite du concours dont ils furent les lauréats. Les 158 logements pour les étudiants de l'École de commerce sont répartis en 112 simplex et 46 duplex dans deux barres parallèles. Ce dispositif, qui apparaît dès 1966 dans l'agglomération bordelaise pour un ensemble d'habitations (Le Grand-Caillou, par André Conte et Georges Prymersky, Eysines), retrouve une nouvelle vigueur dans les années quatre-vingt (Jean Nouvel, Nemausus I, 1985-1987, Nîmes ; 1987-1991, Renzo Piano, logements de la rue de Meaux, Paris) en valorisant l'espace intermédiaire qui acquiert une valeur d'usage importante et devient un lieu tranquille et convivial. Dans la résidence Campus, les longues barres de cinq niveaux sont parcourues de coursives reliées les unes aux autres par des passerelles enjambant un jardin et un petit ruisseau. À l'instar de Nemausus I, le bardage d'aluminium de ces coques en béton accentue la métaphore navale qui transforme la chambre en cabine éclairée par des hublots, l'immeuble en navire et le séjour de l'étudiant en un voyage entre deux rives. Le corps du bâtiment nord est entièrement traversé sur deux étages par un grand vide rectangulaire qui accueillait au départ un petit jardin laissant entrevoir les travées rouges et jaunes de la barre opposée. On sera sensible ici à l'hommage rendu à Le Corbusier mais aussi au clin d'œil adressé à l'immeuble Atlantis du groupe Arquitectonica (Miami, 1980-1982), très médiatisé à cette époque et bien connu à Bordeaux grâce à l'exposition d'Arc-en-Rêve consacrée à l'agence américaine (28 avril-17 juillet 1988). La volonté des maitres d'ouvrage (Domofrance) de financer des concepts architecturaux innovants propres à produire des dispositifs qui séduisent par leur dynamisme formel et spatial renouvelant une typologie, en l'occurrence le logement étudiant, d'un intérêt architectural généralement limité se manifeste à nouveau dans la résidence Claude Ferret conçue par Alain Loisier (1996) comme une couronne ou un cirque de quatre niveaux traversé par une tranchée qui ouvre une percée visuelle vers le bois de Thouars et l'École d'architecture.

(fig. $\left.n^{\circ} 12\right)$ 


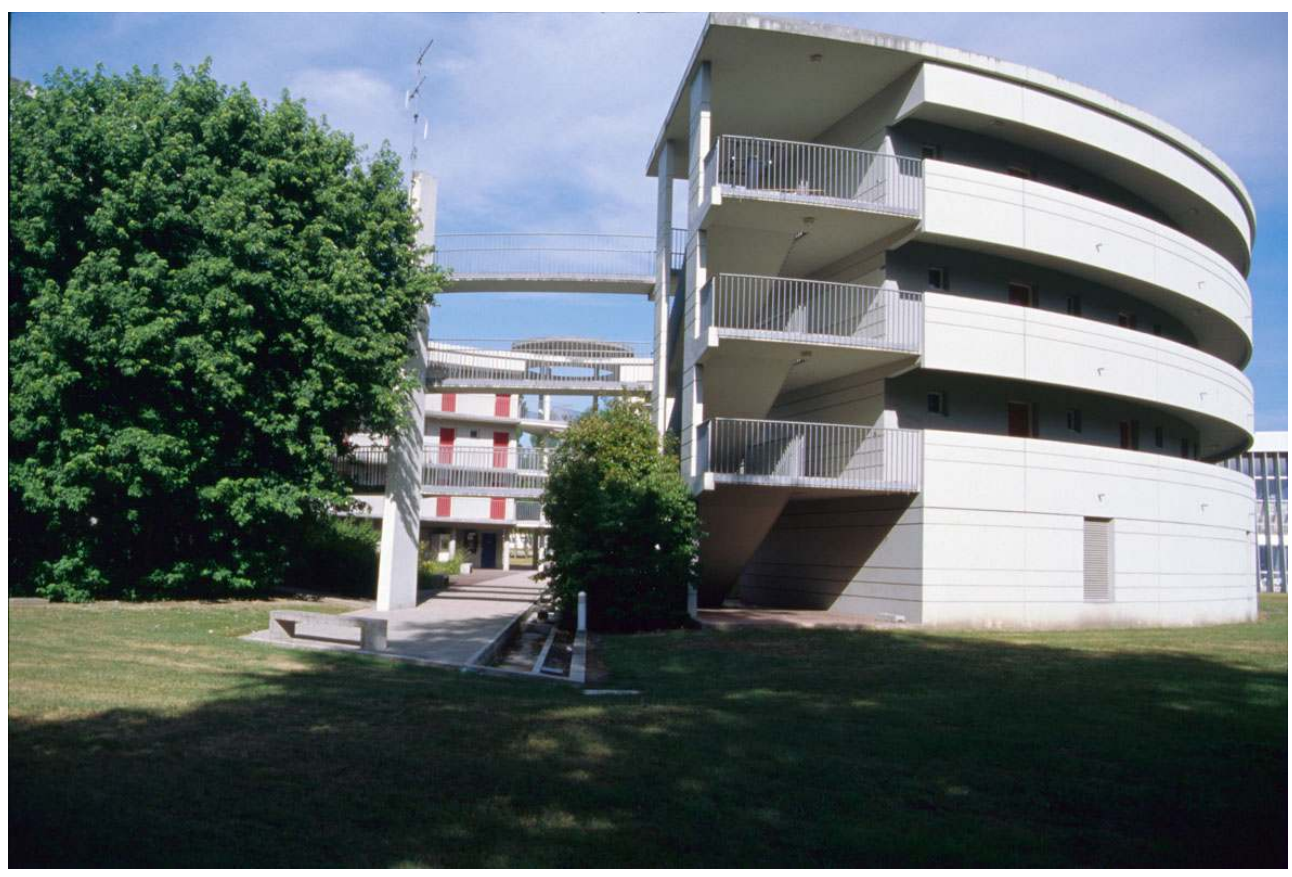

Alain Loisier, Jean de Giacinto : résidence Claude-Ferret, Talence, 1996.

(C) Marc Saboya.

Les bâtiments de l'École subissent eux aussi des modifications et pour cela le directeur de l'époque, Vincent Auzanneau, souhaite mettre à contribution de jeunes architectes fraîchement diplômés. En 1991, Bernard Murua et Gilles Dubardier réalisent une extension à la bibliothèque en forme de vaisseau de métal aux façades biaises. En 1994, Karine Louillot et Pierre Goutti achèvent leur première grande commande publique, le centre informatique audio et vidéo (C.I.A.V.) qui doit faire partie d'un plan de restructuration de l'école que les deux architectes ont dessiné dès $1992^{24}$. Diplômés depuis 1988 sur un projet de centre de production audiovisuel à Bordeaux-Lac, le programme leur convient. Sensibles à la leçon de Mies van der Rohe, formés chez Hondelatte à l'époque du tribunal de Bordeaux et de ses façades translucides, les deux jeunes architectes réalisent un bâtiment aux lignes rigoureuses et pures planant au-dessus d'un sol de fins pilotis tandis que l'escalier oblique en béton pénétrant brutalement dans le sol surélevé évoque les accès au C.E.T.E. de Saint-Médard-en- Jalles (Hondelatte, 1972). La façade sud est presque aveugle mais au nord, un mur-rideau de verre occupe toute l'élévation et renvoie à un illustre modèle pour une école d'architecture, le Bauhaus de Dessau (1926, W. Gropius). Cette rigueur élégante adaptée à un fonctionnement très simple (la climatisation naturelle par exemple) imposé par des moyens financiers limités affronte la gratuité formelle des courbes de Ferret. En 2003, Jean de Giacinto poursuivra ce parti en prolongeant le C.I.A.V. par un bâtiment d'enseignement et de recherche de volumétrie identique mais sans pilotis et pourvu, sur la façade ouest, de panneaux composites (fibre de verre et polyester) décorés par impression photographique de silhouettes de peupliers d'Italie ${ }^{25}$.

Le parti qui se dessine en 1994 avec le bâtiment de K. Louilot et P. Goutti initie, sur le campus, une tendance vers une économie des moyens dépassant l'enseignement de Jacques Hondelatte pour rencontrer plus largement un courant qui apparait en France 
dans les années quatre-vingt-dix et qui privilégie une forme d'immatérialité, une volumétrie simple, parfois une certaine froideur qui ne dédaigne pas les clins d'œil au style international. Cette tendance a produit, sur le campus, des œuvres singulières qui entretiennent une relation complexe avec un environnement dans lequel elles tentent souvent de se fondre.

Deux réalisations de l'agence Brochet, Lajus et Pueyo nous paraissent de ce point de vue, mais à des degrés divers, intéressantes. En dépit de la légèreté et de l'effet aérien produits par la construction sur pilotis, l'Institut de chimie de la matière condensée-C.N.R.S. (1995, angle avenue d'Ausone et allée Pascal) relève encore d'un certain formalisme ${ }^{26}$. L'immense plate-forme aux murs de verre et bardage métallique est en lévitation audessus d'un vaste parking ponctué la nuit de points lumineux bleus. Des escaliers partant du parking permettent de monter sur ce plateau surélevé où sont installés les laboratoires et où travaille, sur le même niveau, la communauté des chercheurs. La plateforme vient s'appuyer sur une aile en bardage de bois qui met en relation l'auditorium et l'administration. Le jeu sur le léger et le lourd, sur la transparence et la fermeture, sur le matériau industriel (fer et verre) et naturel (bois) produit un ensemble original, éclectique dans l'évocation mêlée de Le Corbusier et Jean Nouvel. Dans le même esprit Henri Blanchot construit l'Espace $5 \mathrm{D}$ à Bordeaux II, une plate-forme avec bardage de pare-soleil en bois portée par des pilotis libérant ainsi un parking (1999).

Figure 13

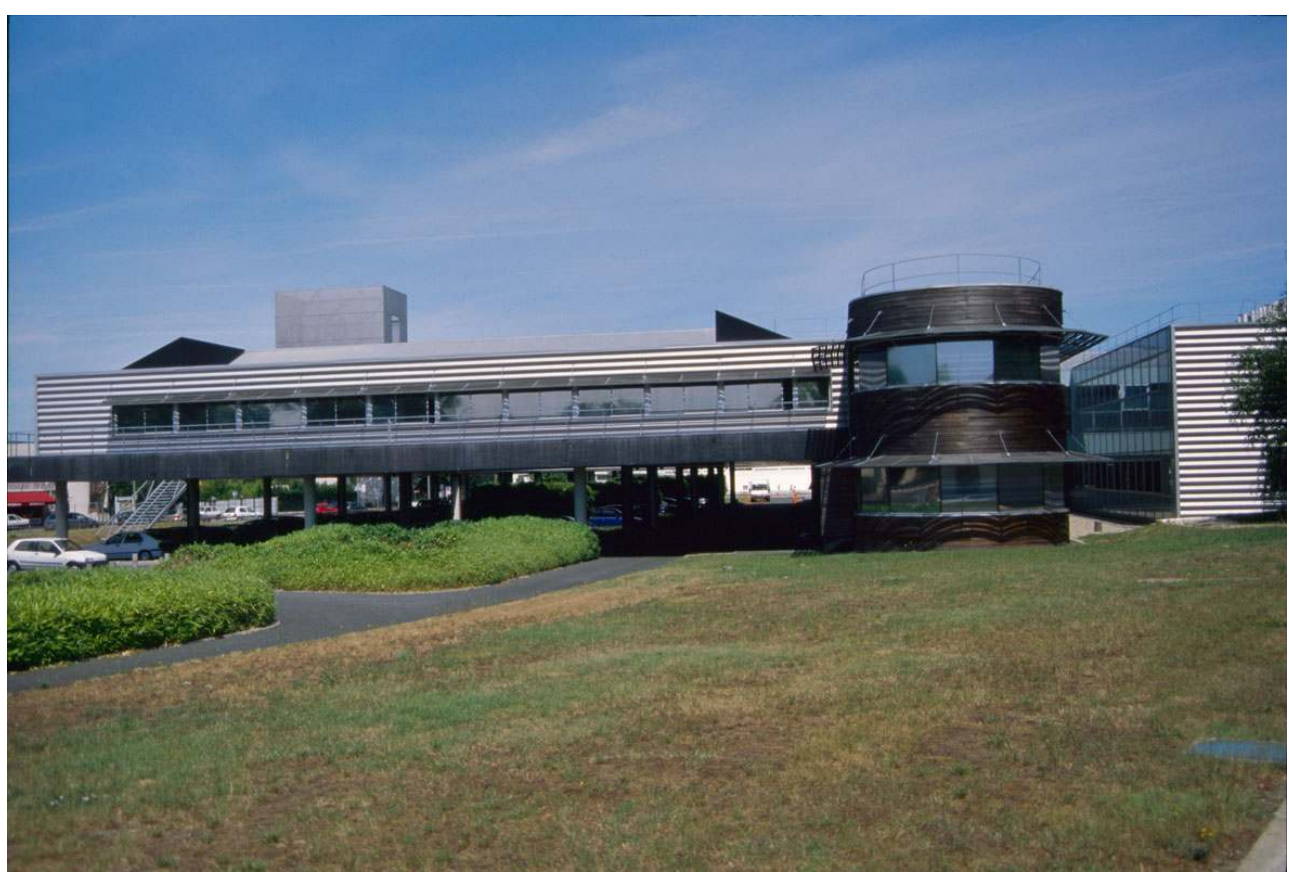

Olivier Brochet, Emmanuel Lajus, Christine Pueyo : institut de chimie de la matière condensée, CNRS, Talence, 1995.

(C) Marc Saboya.

26 Un pas est franchi vers une neutralité plus marquée lors de la construction en 1997 de l'extension de la faculté de droit et de sciences économiques sur le flanc sud du bâtiment - alors que Sainsaulieu prévoyait à l'origine les agrandissements au nord, un emplacement désormais réservé aux espaces verts protégés. Cette situation pose aux 
lauréats du concours le problème de l'installation d'une construction dans un plan masse qui ne l'attendait pas là. Brochet, Lajus et Pueyo conçoivent alors deux pavillons dans l'axe de l'Aula magna. Pour respecter l'échelle du bâtiment d'origine ils enterrent les salles de cours dans l'espace séparant les deux volumes en les éclairant par une vaste fosse ouverte dans le sol et aménagée en jardin à forte pente. Une galerie vitrée, elle aussi enterrée, fait la liaison entre les deux parties. Afin de «minimiser l'impact du projet sur le site» les architectes adoptent "une logique minimaliste»: simplicité et pureté des parallélépipèdes, utilisation d'un seul matériau, le métal déployé, devant un mur de verre qui enveloppe une structure de béton invisible de l'extérieur. Le bardage de fer - ou " râpe à fromage » comme le nomment les étudiants - se soulève par endroits, à la manière des panneaux de J. Nouvel à Bouliac, pour donner des vues sur le campus. Soucieux de conserver le parti de transparence de la galerie de l'ancien édifice, ils élèvent au rez-dechaussée leurs boîtes métalliques sur le socle de verre si bien que le mur de métal, dans le prolongement exact de la jupe vitrée, semble ainsi suspendu dans le vide. La netteté des lignes, la clarté des volumes, le jeu simple et efficace de la lumière sur deux matériaux qui parviennent à s'effacer confinent à l'abstraction et placent cette œuvre sophistiquée, livrée en 1997, parmi les belles réussites du courant minimaliste ${ }^{27}$. Éviter de faire du bâtiment un objet, s'empêcher d'imposer une forme pour s'intégrer au contexte peut conduire à l'enfouissement complet de l'édifice. C'est le parti que choisit Jean-Philippe Pargade en réalisant l'extension de la bibliothèque universitaire droit et lettres (1997-2000). La grande salle de lecture entièrement enterrée sous le bâtiment de L. Sainsaulieu et P. Daurel lui offre un socle immense et reste lisible extérieurement par une bande vitrée qui émerge du sol et fait le tour du nouvel espace.

\section{(fig. $\left.n^{\circ} 14\right)$}

Figure 14

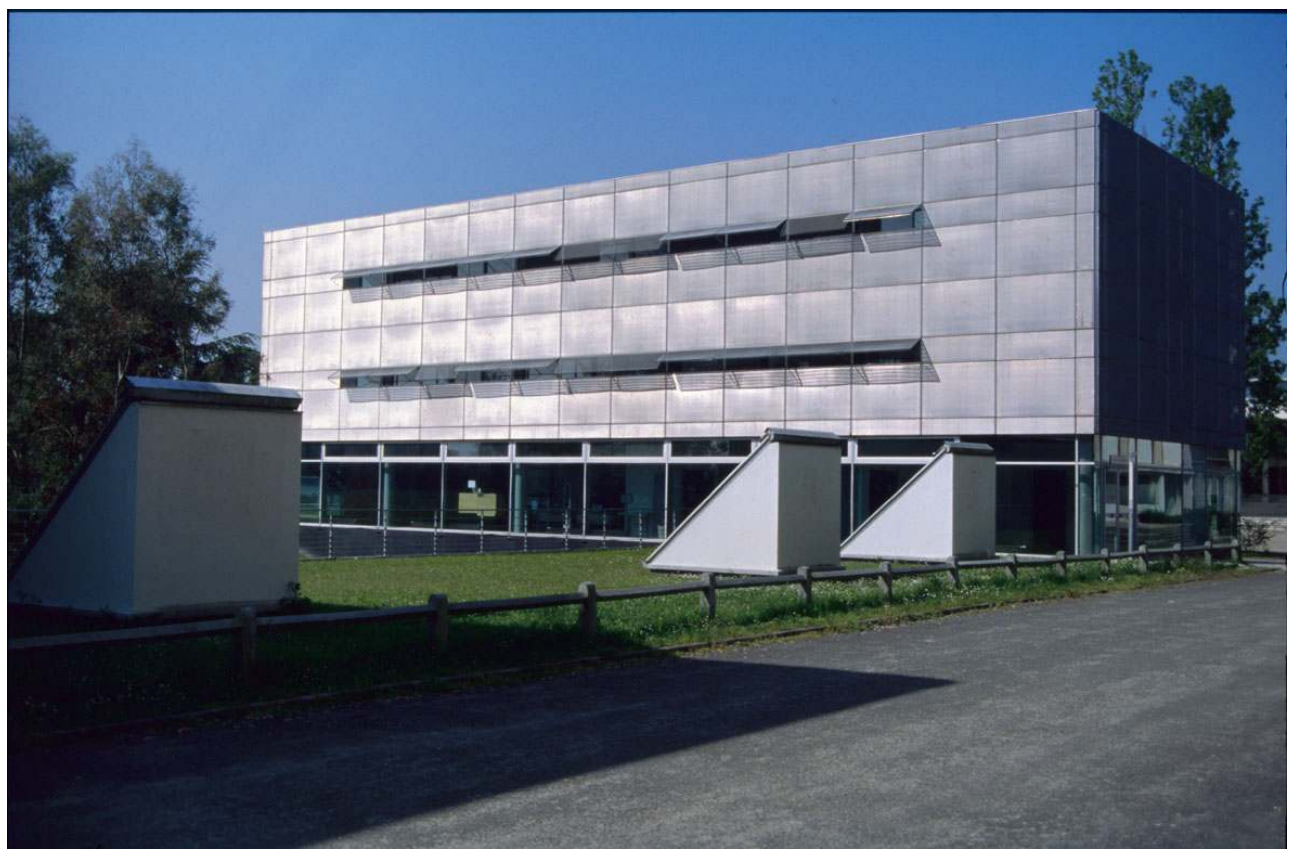

Olivier Brochet, Emmanuel Lajus, Christine Pueyo : extension de la faculté de droit, Pessac, 1997.

(c) Marc Saboya. 
«Faire image », s'imposer comme objet, occuper largement le site et retrouver le sens du monumental voilà les grands principes de la seconde tendance qui affronte sur le campus le courant de la neutralité sophistiquée. Cette orientation est en partie liée au processus permanent de compétition qui conduit les architectes s'affrontant dans les concours à produire des images fortes, spectaculaires pour séduire le jury. Lauréate du concours pour la Maison de l'archéologie, Brigitte Gonfreville reconnaît volontiers que, dans cette course à l'image, sa proposition pour ce projet était la plus percutante. Posé sur un long mur de marbre incliné un immense volume tronconique loge la bibliothèque, espace fermé à l'extérieur, replié sur lui-même où se conserve et se dépose le fruit des travaux des chercheurs, rotondité protectrice débordant sur le monde mais aussi lieu d'une intense incubation. À l'une de ses extrémités le mur s'interrompt pour révéler une structure orthogonale qui quadrille les strates d'une coupe archéologique en résine synthétique éclairée la nuit (œuvre du plasticien Walter Notz). L'unique passage ouvert dans le mur évoque la porte d'un temple. On y accède au prix d'une lente ascension sur un long caillebotis en pente qui conduit à quatre pylônes dont les sommets aigus perturbent la belle horizontalité des lignes. Au-delà de l'écran opaque du mur protecteur $(120 \mathrm{~m}$ de long, $4 \mathrm{~m}$ de haut) qui se dresse tel une enceinte sacrée exhumée des sables de Mésopotamie, les espaces clairs des salles de cours et des laboratoires donnent une autre image de l'archéologue, plus proche du savant que de l'aventurier. Avec cette œuvre livrée en 1992, Brigitte Gonfreville retrouve le plaisir du dessin - «dessiner n'empêche pas de penser " dit-elle en réponse à J. Hondelatte - et la puissance du parti dicté par une bonne analyse du programme, deux points sur lesquels se fondait l'enseignement de son maître Claude Ferret en 1965 : «Ferret nous apprenait à analyser un programme et à en sortir un parti fort, à tirer un parti original, pas le projet de tout le monde $e^{28} »$.

Figure 15

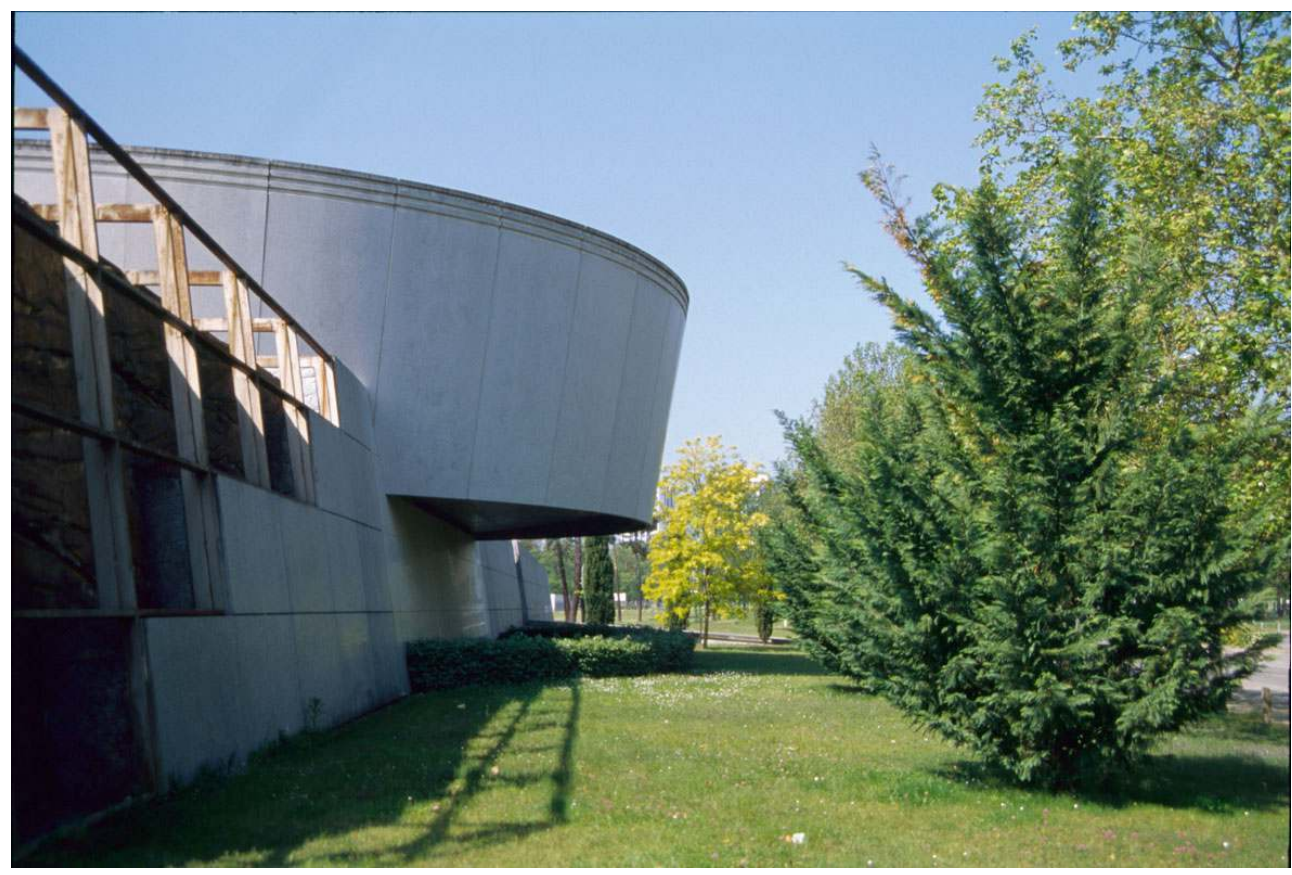

Brigitte Gonfreville-Dumon, Luc Arsène-Henry Jr., Alain Triaud : maison de l'archéologie, Esplanade des Antilles, Pessac, 1992.

(c) Marc Saboya. 
Cette version contemporaine de l'architecture «parlante » héritée de la culture «BeauxArts " annonce des réalisations où la symbolique formelle est très forte. Décrivant son projet pour l'École nationale supérieure d'électronique et de radioélectricité de Bordeaux (E.N.S.E.R.B., rue Albert-Schweitzer, Talence) achevée en 1995, Luc Arsène-Henry précise : "L'identité visuelle du bâtiment est forte, résolument fulgurante et avant-gardiste. (...) Vaisseau accueillant posé à la frontière du domaine universitaire, il tourne sa coupole à l'écoute du cosmos ". Si l'Institut de chimie-C.N.R.S. tout proche plane au-dessus du sol, l'E.N.S.E.R.B. prend possession du territoire en étendant ses ramifications aux formes douces du relief. Grande aile blanche greffée à un long fuselage étroit percé de hublots, le "vaisseau » futuriste habité épouse l'ondulation de la butte où il s'est posé. «Lieu de vie, village où tous les espaces, les cheminements sont prétextes à la rencontre (...) à l'échange fertile des connaissances et des intuitions ${ }^{29}$ ", il est, en outre, l'image de la sophistication technologique. Cette adaptation au relief anime en fait un plan très simple en peigne. Le long et haut fuselage abrite une vaste rue intérieure en pente légère éclairée par des lampadaires. Elle distribue des bureaux, des salles de cours et trois ailes au nord dont la longueur décroît vers l'ouest selon la forme triangulaire du terrain. Celles-ci sont unies extérieurement par une grande structure de béton qui condense en une ligne souple et plastique tout le mouvement sous la forme d'une oblique dynamique. Entre chaque aile s'ouvrent des patios partiellement abrités par une treillage métallique tandis que de gros canons de lumière éclairent les amphithéâtres souterrains. Au sud, la masse sombre, lisse et fermée du grand amphithéâtre s'enfonce dans la terre tel une météorite ou un lourd rocher poli. Proche de la faculté de droit, le centre Condorcet, bel objet rond et vitré au toit incliné, développe une intéressante rue intérieure qui scinde le bâtiment avec franchise (Archigroup, 1993). Près de l'E.N.S.E.R.B., sur l'emplacement de l'ancien stadium universitaire réalisé par Jacques d'Welles, Franck Hammoutène achève en 2003 l'Institut européen de Chimie et de Biologie (I.E.C.B.), un projet sans équivalent en France. Depuis la rue Schweitzer l'édifice offre ses trois séquences parallèles soulignées par de grands écrans de béton nets, francs, débordant du volume général et contrastant avec la courbe plastique de l'auvent blanc d'entrée à l'ouest. Au nord, une butte de terre rapportée enveloppe les salles de résonance magnétique. Entre Le Corbusier et Richard Meier l'œuvre de l'architecte parisien, auteur de la Z.A.C. de Bercy et de l'église Notre-Dame-dela-Pentecôte (Paris-La Défense), affiche un style « à la fois référencé et éminemment personnel (...) qui confirme la force et la justesse d'un style conscient de son héritage ${ }^{30}$ ». (fig. $\left.n^{\circ} 16\right)$ 


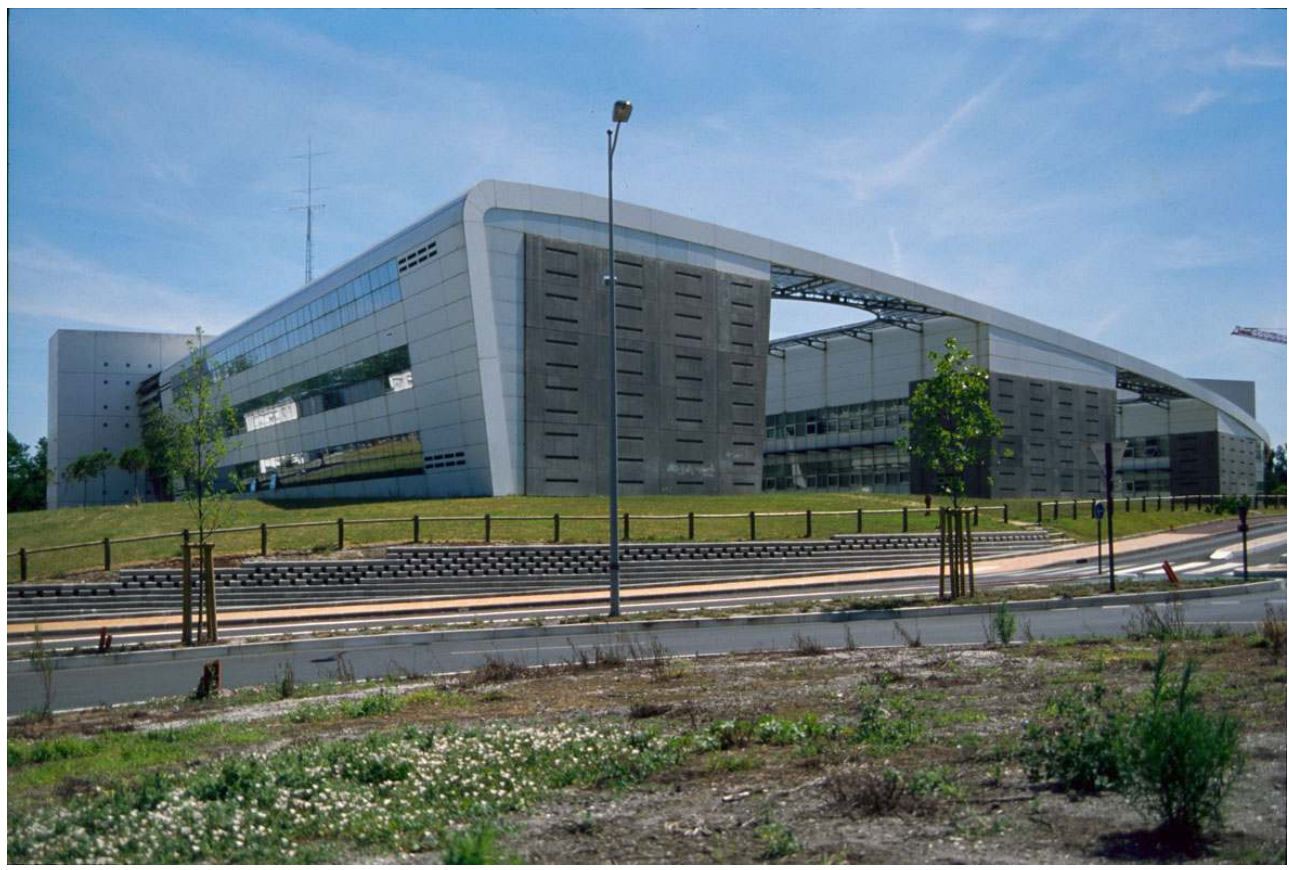

Luc Arsène-Henry Jr, Alain Triaud : ENSERB, Talence, 1995.

(c) Marc Saboya.

Autre image forte, la Maison des arts de Massimiliano Fuksas, achevée en 1995 (esplanade des Antilles, Talence). Le choix après concours de l'architecte italien, résidant à Paris depuis 1989, révèle la tendance qui se développe partout en France à la fin des années quatre-vingt d'accueillir des œuvres de qualité réalisées par des bâtisseurs de renommée internationale. Pour la première fois de son histoire le pays s'ouvre largement aux plus grands noms de l'architecture mondiale : M. Botta, K. Tange, A. Rossi, R. Piano, R. Meier, K. Roche, W. Alsop, R. Bofill, S. Calatrava, O. Bohigas, F.O. Gehry. Certains, tel R. Koolhaas, N. Foster, R. Rogers et M. Fuksas atteignent même Bordeaux. Long et immense monolithe de cuivre vert posé à même le sol, la Maison des arts est un signal au sein du campus, d'abord par sa couleur agressive ensuite par son gabarit hors d'échelle, enfin par son parti de fermeture. Fractionnée par des déchirures de verre, saignées verticales et entaille horizontale, sa précieuse peau de cuivre oxydé acquiert, peu à peu, une patine délicate et nuancée. La fissure horizontale qui fait le tour du bâtiment et fait rentrer la lumière met le couvercle de ce conteneur des arts en lévitation comme s'il était soulevé par la puissance créatrice qui bouillonne au sein de l'édifice. Sur le toit, une fragile galerie ouverte conduit à une autre boîte - le siège de radio-campus - plus petite et en bois débordant largement sur la façade nord, un surplomb audacieux que l'on retrouve parfois dans l'art de Fuksas et qui est destiné à briser un rythme, casser un volume, bouleverser une échelle (1992-1999, îlot Candie-Saint-Bernard, Paris). À l'intérieur règne partout le béton brut de décoffrage qui n'impose pas pourtant une banalisation des espaces. Les deux immenses halls traversants conduisent à des salles étudiées pour accueillir des activités variées : cinéma, musique, arts plastiques, théâtre (salle de 350 places). Certains utilisateurs pensent tout de même que l'édifice est fonctionnellement raté et techniquement précaire mais les œuvres les plus radicales ont toujours suscité les critiques les plus sévères et on connaît chez Fuksas son sens développé pour la 
provocation : "La première chose que doit faire un architecte c'est de ne pas lire le programme. Sinon il ne gagne pas le concours ». (fig. $\left.\mathbf{n}^{\circ} \mathbf{1 7}\right)\left(\right.$ fig. $\left.\mathbf{n}^{\circ} \mathbf{1 8}\right)$

Figure 17

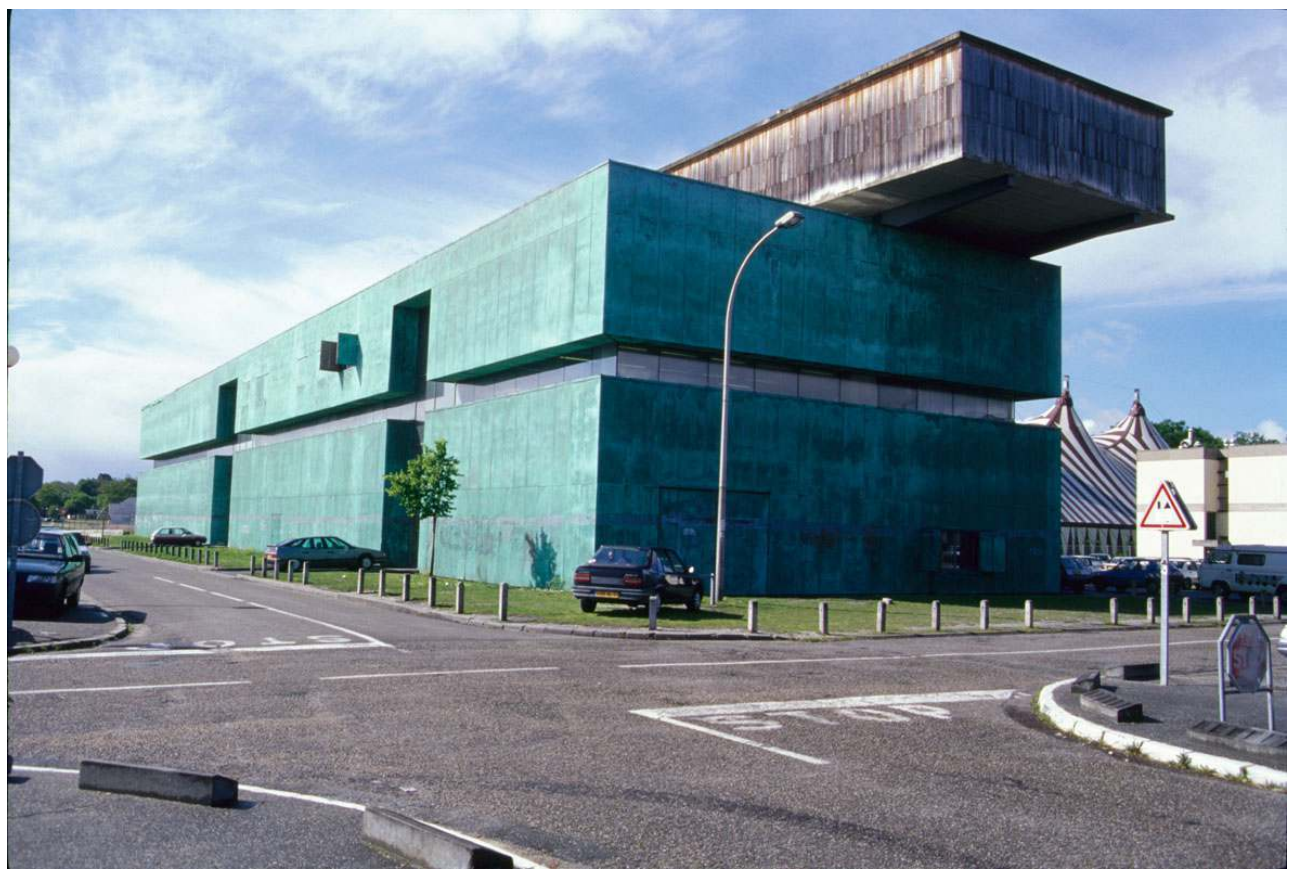

Massimiliano Fuksas : maison des Arts, esplanade des Antilles, Pessac, 1995 (c) Marc Saboya.

Figure 18

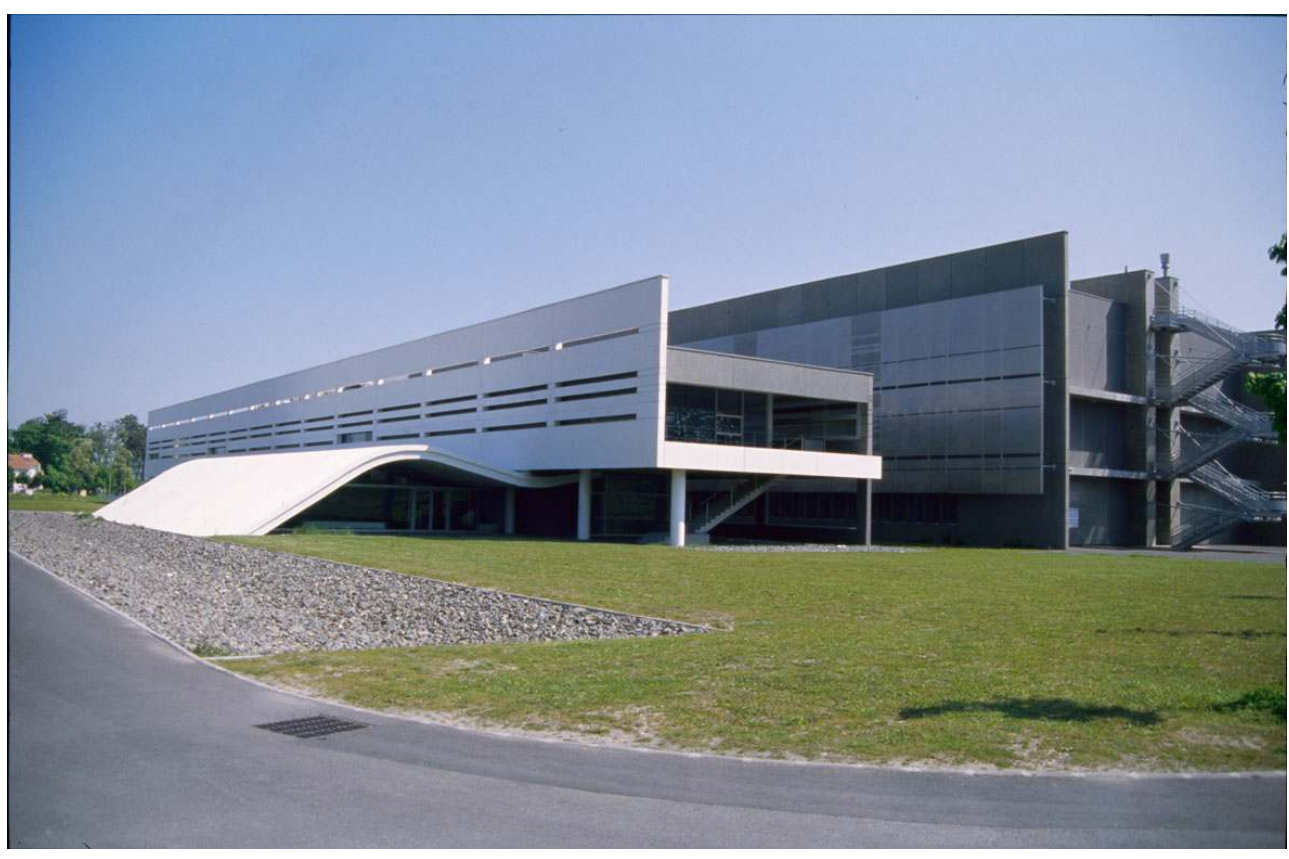

Franck Hammoutène : institut européen de chimie et de biologie, Pessac, 2003.

(c) Marc Saboya. 
Pas de provocation ni de caprice de star chez Nicolas Ragueneau et Antoine Roux qui interviennent surtout sur le domaine universitaire de Bordeaux II, mais un grand professionnalisme hérité de leur passage (près de dix ans pour Ragueneau) dans l'agence de Claude Bouey. Collaborateur de Pierre Mathieu depuis 1953 et son associé depuis 1960 aux côtés de Bersagol, Tagini et Sérieis, Bouey avait hérité du secteur hospitalier lors de l'éclatement de l'atelier ${ }^{31}$. Diplômé en 1982, N. Ragueneau apporte une approche technique appuyée - en particulier dans le domaine de l'acier - acquise à l'École d'architecture de Tounay (Belgique). C. Bouey, A. Roux et N. Ragueneau entreprennent à Bordeaux II d'importants travaux qu'ils souhaitent inscrire dans la continuité de l'œuvre de Mathieu, des bâtiments nouveaux mais respectueux d'un plan d'urbanisme cohérent et d'une architecture de qualité. Ce parti d'intégration tranche avec la recherche de la singularité qui caractérise le campus de Talence-Pessac. Certes, il est imposé par la superficie réduite dont dispose le C.H.U. mais il est aussi guidé par une certaine humilité de la part des architectes et par la conscience d'être les héritiers d'un atelier prestigieux. C'est dans cet esprit que s'inscrivent Ragueneau et Roux, continuateurs de l'esprit d'une agence et non d'une école, d'où le caractère un peu atypique de leur production plus internationale dans ses choix esthétiques que la production bordelaise contemporaine. À Bordeaux II, les architectes entendent ainsi révéler le site et non pas le violer, continuer sa construction et non pas la brusquer. Ils bannissent ainsi la diversité des matériaux et des couleurs mais ne refusent pas la quête d'une image identificatrice et s'intègrent ainsi dans la tendance monumentale que nous traitons ici. «Une modernité dans une forme de continuité » dit $\mathrm{N}$. Ragueneau pour définir le travail de l'équipe à Bordeaux II ${ }^{32}$. L'extension de la bibliothèque universitaire le confirme. Au bâtiment brutaliste de Mathieu, Claude Bouey et ses deux associés greffent une rotonde qui prolonge, à chaque niveau, les plateaux existants, fonctionne en continuité directe avec la bibliothèque et s'inscrit par sa forme dans la logique volumétrique du modernisme ${ }^{33}$. La touche contemporaine est dans le revêtement en tôle perforée d'aluminium laqué habillant le cylindre. 


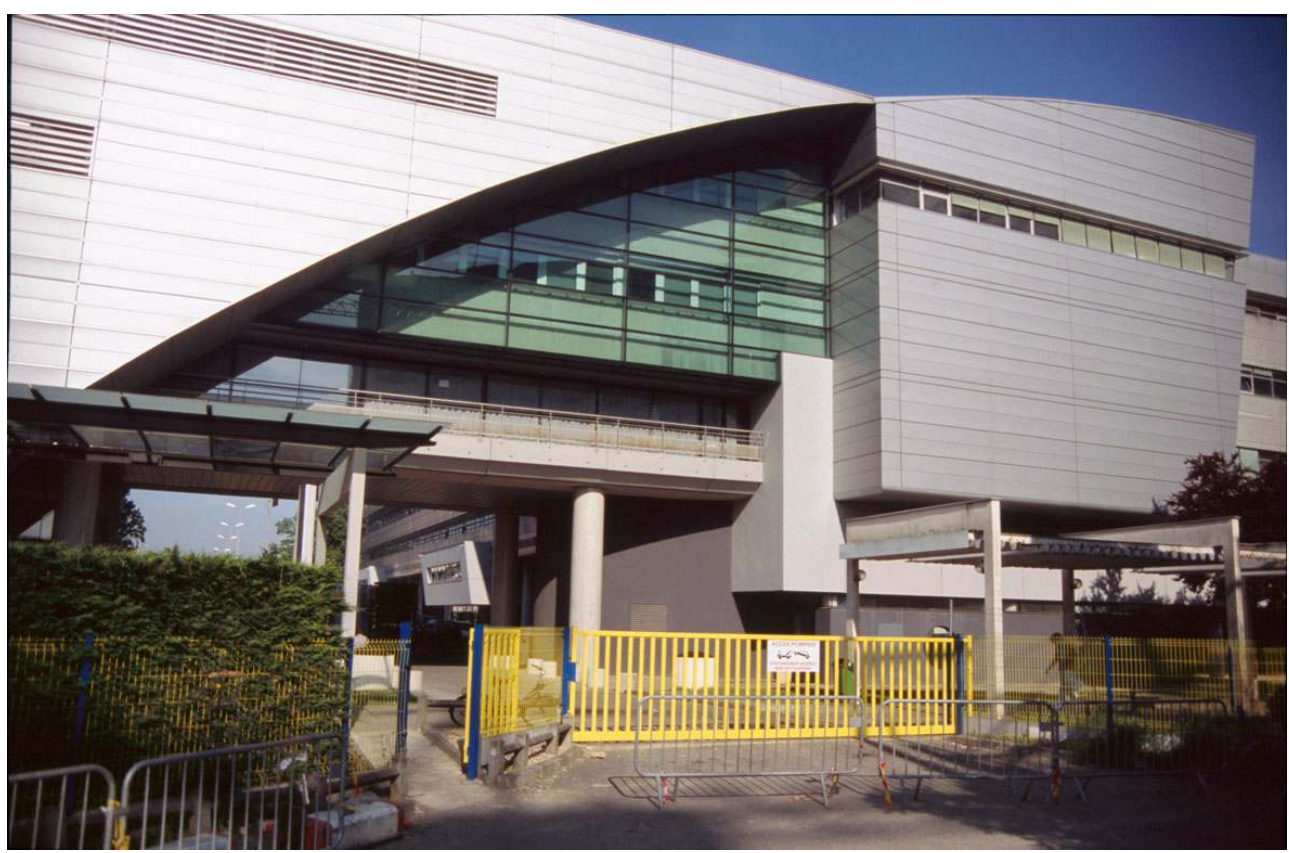

C. Bouey, N. Ragueneau, A. Roux : centre de pharmacie, CHU-Pellegrin, Bordeaux, 1992 et 2002.

(c) Marc Saboya.

En 1992, la construction du centre de pharmacie, complété en 2002, reprend la disposition en peigne du plan de Mathieu en prolongeant deux ailes de la composition d'origine et en doublant la grande barre. À l'est, le centre est longé par une galerie couverte d'une verrière incrustée de photographies originales de Ferrante Ferranti ${ }^{34}$. À l'ouest, le bâtiment se présente sous la forme de deux hautes et longues barres dessinant un plan en L. L'angle est marqué par un mur-rideau de verre en segment de cercle à l'endroit où s'interrompt la peau métallique qui, avec les hublots, les coursives, l'immense pare-soleil en guise de corniche, le jeu sur la variation des matériaux et le passage à une texture plus lisse assure, selon N. Ragueneau, une "cohabitation sereine» avec l'architecture de Mathieu. En revanche, l'École supérieure de biotechnologie des molécules (Ragueneau et Roux, 2000) située aux confins du campus et sur une hauteur, donc décentrée par rapport au C.H.U., est traitée dans une logique d'image forte semblable aux réalisations de Bordeaux I et III mais conforme à la demande du directeur fondateur de l'école, le professeur Claude Cassagne. La grande sphère de l'amphithéâtre en métal bleu est un signal dans le domaine universitaire et traduit aussi une certaine autonomie de l'institution au sein du campus.

(fig. $\left.n^{\circ} 20\right)$ 


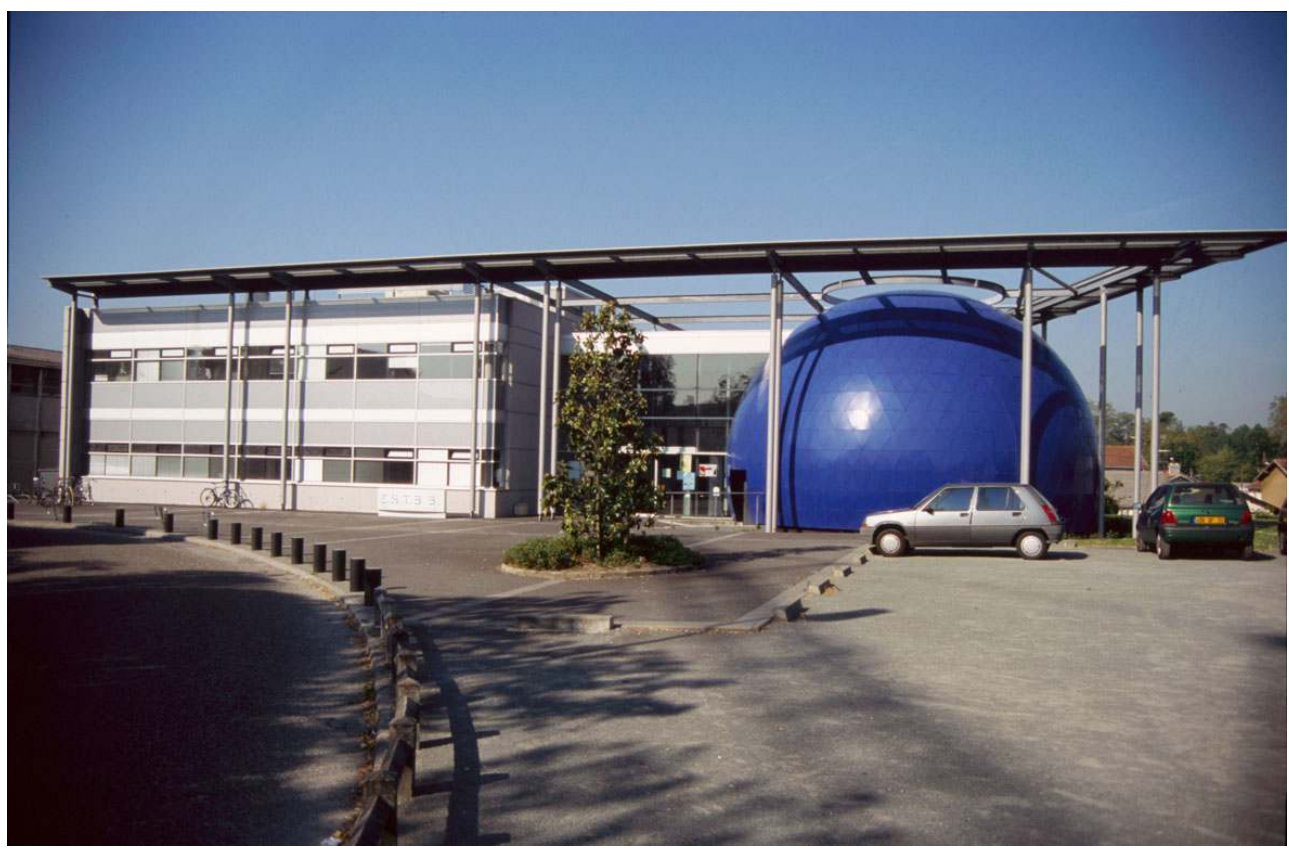

N. Ragueneau, A. Roux : école supérieure de biotechnologie des molécules, CHU-Pellegrin, Bordeaux, 2000.

(c) Marc Saboya.

\section{NOTES}

1. - Cet article est, pour l'essentiel, extrait de l'ouvrage de : COUSTET, Robert et SABOYA, Marc. Bordeaux, la conquête de la modernité: Architecture et urbanisme à Bordeaux et dans l'agglomération de 1920 à 2003. Bordeaux : Mollat, 2005.

2. - Les $4 / 5^{\mathrm{e}}$ de la superficie du campus sont réservés aux espaces verts et terrains de sport. Dans ses premiers projets, Sainsaulieu prévoyait une installation sportive pour chaque faculté. La répartition adoptée permet une meilleure distribution du domaine.

3. - Les ateliers "lourds» (usinage, fonderie, etc.) et la chaufferie sont communs aux deux établissements.

4. - Évolution humaine des origines à nos jours, 1968-69.

5. - La cour en U des lettres affrontait ainsi l'espace ouvert du parvis de droit. Depuis la construction des deux bâtiments de Brochet, Lajus et Pueyo, l'esplanade de droit adopte désormais un plan en $\mathrm{U}$ tandis que la construction du bâtiment d'accueil des étudiants ferme la cour des lettres.

6. - Pour les différents plans masse, voir fonds Daurel, 3 U 9-14. Ces patios ont été, par la suite, investis par des bâtiments annexes. Les parois vitrées des amphithéâtres ont été murées et le parti architectural adopté par P. Mathieu jouant sur la transparence et l'alternance patio/ architecture est profondément dénaturé par ces ajouts. 
7. - JULLIAN, René. Histoire de l'architecture moderne en France, de 1889 à nos jours. Paris : Philippe Sers, 1984, p. 270.

8. - J. Sérieis fait l'unanimité et S. Botarelli est apprécié. En revanche, la majorité des architectes rencontrés s'accordent à reconnaître le peu de tact dont fait preuve Y. Salier, bon patron dans son agence mais pédagogue contesté à l'École. Ceux qui, tel Alain Charrier, apprécient ses qualités d'enseignant admettent avoir surtout appris en travaillant dans l'agence de Mérignac.

9. - Entretien avec Piou Lacoste, 11 mai 2003.

10. - Selon J.M. Billa, les $2 / 3$ des effectifs quittent l'École à la rentrée 1969-1970 pour aller à Paris (U.P. 6), Toulouse, Nantes et Grenoble (entretien du 6 octobre 2003).

11. - C. Laroche, entretien du 16 juillet 2003.

12. - L'ouvrage de Bernard Rudofsky, Architecture without architects, (1964), connaissait alors un grand succès auprès des architectes désireux de trouver une alternative aux principes académiques et aux positions doctrinaires du Mouvement moderne. Piou Lacoste, entretien du 11 mai 2003.

13. - Entretien du 3 juillet 2002.

14. - Ce diplôme, soutenu dans la nouvelle école, sous la pyramide, est certainement le premier diplôme collectif présenté à Bordeaux. Le directeur-administrateur de l'école refusa d'ailleurs d'assister à la soutenance qui eut lieu devant un jury composé de P. Lajus, P. Anus, et Charbonneau.

15. - Entretien avec Ch. Maudet, 21 octobre 2004.

16. - Les modifications intérieures engagées en 1996 par Christian Bardin, Christophe Bouriette, Isabelle Mathieu, Aline Rodrigues et Laurent Vilette ont redonné à la pyramide ses qualités de lieu de convivialité (voir A.M.C., 52-53, juin-juillet 1994).

17. - RAGOT, Gilles, MONTHIERS, Vincent, JACQUES, Michel (dir.). Guide d'architecture. Bordeaux et son agglomération, 1945-1995. Bordeaux : Arc en rêve-Centre d'architecture, 1996, p. 203.

18. - ALVARO, Johann. L'enseignement de Claude Ferret à l'école d'architecture de Bordeaux: un enseignement de maître d'atelier, 1942-1977. Mémoire de $2^{\mathrm{e}}$ cycle, École d'architecture de Bordeaux, 2000-2001. L'interview de Pierre Lajus citée dans ce travail date du 15 mai 2001. Serge Botarelli : «Claude Ferret est pour moi ce que le cinéma muet est pour le cinéma parlant » et Brigitte Gonfreville : « Ferret m'a tout appris. Il m'adorait » (entretien du 5 juillet 2002).

19. - RAGOT, Gilles, MONTHIERS, Vincent, JACQUES, Michel (dir.). Guide d'architecture. Bordeaux et son agglomération, 1945-1995. Bordeaux : Arc en rêve-Centre d'architecture, 1996, p. 124.

20. - Dans le plan masse directeur d'octobre 1969, un second tripode est prévu à l'extrémité ouest du domaine (fonds Daurel, 1 U 6 105. Plan reproduit dans: SAVÈS, Véronique. Les deux domaines universitaires de Bordeaux. T.E.R. Histoire de l'art contemporain, Université Michel de MontaigneBordeaux III, 1992, t. 2, pl. 134). Il ne sera pas construit.

21. - SABOYA, Marc. "Anamorphose urbaine». Le Festin, ${ }^{\circ} 46,2003$, p. 114. Jean Sérieis (1920-1981) a une part importante dans la construction du tripode. Élève de Lecomte (Paris) et de Ferret (Bordeaux), il est logiste et diplômé en 1952. Il réalise le tripode, les écoles de sagesfemmes, d'infirmières, le centre de transfusion sanguine.

22. - A.A., n¹13-114, avril-mai, 1964, cité par LUCAN, Jacques. "Architecture en France ». Le Moniteur, Paris, 2001, p. 97.

23. - Voir le très intéressant article: DESMOULINS, Christine. «Plan Université 2000. La décentralisation à l'œuvre ». Architecture d'intérieur, Créé, n² 273, 1996, p. 32-42.

24. - Projet en association avec Jacques Robert. La restructuration sera abandonnée par la suite (archives des architectes).

25. - Le C.I.A.V. de K. Louilot et P. Goutti comportait une discrète mise en lumière par l'installation de fibres optiques qui, plus tard, ont grillé et n'ont pas été remplacées. Le projet de J. de Giacinto a été choisi après concours car le nouveau directeur de l'École, Jean-Marc Cailleau entendait renouer avec cette procédure. Les peupliers de Giacinto reprennent la haie de 
peupliers plantée parallèlement à la façade du bâtiment (CLEMENCEAU, Coralie. Matériaux composites et lumières : l'industriel poétique dans l'œuvre de l'architecte bordelais Jean de Giacinto. T.E.R., Histoire de l'art contemporain, université Michel de Montaigne-Bordeaux III, 2003, p. 83-86).

26. - Construit avec Joessel.

27. - Les citations sont extraites d'un entretien avec E. Lajus (10 juillet 2002) qui revendique cette inscription dans le minimalisme reconnue depuis par de nombreux ouvrages d'histoire de l'architecture contemporaine (voir JAQUARD et GODDEFROY. Young French Architects. Birkhäuser, 1999).

28. - Entretien du 5 juillet 2002. Brigitte Gonfreville est diplômée en 1969.

29. - Luc Arsène-Henry, cité par: RAGOT, Gilles, MONTHIERS, Vincent, JACQUES, Michel (dir.). Guide d'architecture. Bordeaux et son agglomération, 1945-1995. Bordeaux : Arc en rêve-Centre d'architecture, 1996, p. 197. L'E.N.S.E.R.B.est l'œuvre de Luc Arsène-Henry et Alain Triaud.

30. - COSTEDOAT, D. « De modernité et d'ailleurs ». Le Festin, n46, 2003, p. 98-99.

31. - Claude Bouey a travaillé à la construction de la faculté de droit et des sciences économiques et de l'I.E.P. Il a réalisé le Centre d'études de géographie tropicale et l'extension de l'Institut d'œnologie.

32. - Nicolas Ragueneau, entretien du 28 juin 2002.

33. Voir RAGOT, Gilles, MONTHIERS, Vincent, JACQUES, Michel (dir.). Guide d'architecture. Bordeaux et son agglomération, 1945-1995. Bordeaux : Arc en rêve-Centre d'architecture, 1996, p. 127.

34. Brochet, Lajus, Pueyo, 2002. Les œuvres photographiées sont des sculptures de Canova, le Bernin, Thorwaldsen, Maderno, Puget, etc.

INDEX

Mots-clés : architecture XXe siècle, architecture universitaire, campus, Mouvement moderne, fonctionnalisme, béton, préfabrication, urbanisme

\section{AUTEUR}

\section{MARC SABOYA}

maître de conférences H.D.R., université Michel de Montaigne-Bordeaux III marcsaboya@hotmail.com 